\title{
Oggetti persistenti La somiglianza a posteriori nelle icone del design
}

\author{
Anna Riboldi \\ Politecnico di Milano \\ anna.riboldi@mail.polimi.it \\ Salvatore Zingale \\ Politecnico di Milano, Dipartimento di Design \\ salvatore.zingale@polimi.it \\ $<$ www.salvatorezingale.it $>$
}

\begin{abstract}
Design has made everyday artifacts less and less anonymous, first recognizing their authorship and then providing them with a title and a name. Some of these artifacts have started to live a "cultural life" of their own, so much so that they are considered far beyond their use performance and regardless of material and technological qualities. They have become icons of their time, similar to the icons of popular culture, to the stars and other celebrities of the cultural industry. Or are they perhaps still similar to the sacred icons of Byzantine art?

This paper first questions why these "everyday objects" are recognized as iconic, and whether there are reasons to consider them "icons" even starting from the semiotic notion introduced by Charles Peirce. Secondly, some industrial products and communicative artifacts of the twentieth century are reviewed, either chosen because they are already recognized as icons, or because they help us better understand this destiny of celebrity that binds them to the values of the era that they have the good fortune to represent.
\end{abstract}

\section{Keywords}

Icon; Design; Likeness; Epochality; Intertextuality

\section{Sommario}

1. Il destino inquieto di un termine fortunato

2. La somiglianza a posteriori

3. Icone del divino e del profano

4. Artefatto iconico e abiti sociali

5. L'icona come carattere

6. I canali alternativi dell'icona

7. Icona, tempo e spazio

8. Icone del design e autore collettivo

9. Conclusione: ci saranno altre icone?

Bibliografia 


\section{Ocula}

Vol 21, No 22 (April 2020) • DOI: 10.12977/ocula2020-21

Anna Riboldi e Salvatore Zingale | Oggetti persistenti. La somiglianza a posteriori nelle icone del design

\section{Il destino inquieto di un termine fortunato}

A volte viene da chiedersi se Charles S. Peirce non abbia scelto il nome sbagliato - Icona - per indicare uno dei tre "tipi di segni", quello relativo ai rapporti tra un segno e il suo oggetto. Così come era accaduto con il termine Pragmatismo, ci sono stati diversi "rapitori di bambini", per usare una sua espressione, che hanno condotto la sua creatura per sentieri diversi da quelli da lui pensati. ${ }^{1}$ Ma sappiamo che le parole hanno anche vita propria, una autonomia storica ed evolutiva; sovrastano la terminologia "alta" e la tradiscono senza scrupolo alcuno: non per ribellione, ma perché la ignorano.

Il termine Icona, più che giustificato per il suo riferimento all'immagine (દikóva), ha infatti subìto applicazioni lontane dall'intento peirceano, ${ }^{2}$ spesso anche fra loro divergenti, seppure tutte fedeli all'etimo. In alcuni contesti il termine sta ancora e sempre a indicare l'immagine sacra dell'arte bizantina e russa, che però ha un omologo profano nelle "iconcine" delle interfacce dei nostri computer. Null'altro accomuna questi due tipi di immagini, se non il fatto di essere immagini.

La società dei media e l'industria culturale hanno poi elevato a icone pop dive e divi dello spettacolo, divenuti così rappresentanti della propria epoca e modelli da seguire nel look o nello stile di vita: da Marilyn Monroe a James Dean, da Elvis Presley ai Beatles (cfr. Spaziante 2016). Analoga sorte hanno avuto l'effige del presidente Mao, specie dopo la variopinta serigrafia di Andy Warhol, e il Che Guevara da T-shirt. Insomma, interpretanti iconici del proprio tempo.

Anche in campo semiotico questo termine ha avuto (e ha) un destino travagliato. Per molti, icona è ancora un sinonimo di "segno visivo", anche se ormai sappiamo che questa accezione è limitativa. In Algirdas J. Greimas poi, in una semiotica tutt'altro che peirceana, dapprima nella voce "Iconicità" del Dictionnaire del 1979 e poi nel saggio Semiotica figurativa e semiotica plastica del 1984, i termini icona e iconicità vengono confinati nello spazio angusto dell'“imitazione della realtà", cui viene affidata la prosaica funzione di «produrre l'illusione referenziale» (Greimas e Courtés 1979: 150, tr. it.).

C'è però un elemento in comune, e non può che essere così, in tutti questi usi: la nozione di somiglianza. Non è questo il luogo per riprendere l'annosa discussione, che ci riporta agli anni Settanta, sull'iconismo e sulla definizione

1 Di questo avviso è, fra gli altri, Emanuele Fadda: «[...] gli usi prevalenti del termine "iconismo" sono ben poco fedeli al dettato peirceano. La nozione viene usata infatti per lo più per riferirsi ad immagini - ma le immagini non sono una classe di icone [...]. La caratterizzazione di icona come somiglianza (likeness) viene poi spesso travisata nel senso di “copia”, sicché l'icona sarebbe tanto più tale quanto più fedele all'originale [...]» (Fadda 2013: 179).

2 Per una trattazione esauriente della nozione di icona in Peirce vedi Proni (2017). Proni sottolinea sia l'ambiguità già presente nello stesso Peirce nell'uso di questo concetto, sia però anche la chiarezza di fondo di questo assunto teorico. E conclude: «Insomma, l'Icona intesa in senso rigoroso è una qualità pura che sta per una qualità pura secondo una relazione possibile per un interpretante» (Proni 2017: 367). Da cui si evince che, insieme a somiglianza, i due caratteri costitutivi dell'icona in Peirce sono qualità e possibilità. 


\section{Ocula}

Vol 21, No 22 (April 2020) • DOI: 10.12977/ocula2020-21

Anna Riboldi e Salvatore Zingale | Oggetti persistenti. La somiglianza a posteriori nelle icone del design

di somiglianza. ${ }^{3} \mathrm{Ci}$ limitiamo ad assumere la somiglianza come quel processo empirico, percettivo e mentale, che ad esempio ci permette di inferire una relazione di parentela confrontando i lineamenti di due persone che non conosciamo ma che possiamo confrontare visualmente. Più in generale, si ha significazione per somiglianza quando in un oggetto riconosciamo proprietà, qualità o tratti pertinenti presenti anche in un altro oggetto, in modo tale per cui il primo diventa significante del secondo. Dove un oggetto può anche essere un'idea o uno stato d'animo: di che cosa è iconico il dipinto L'urlo di Edvard Munch, di un uomo che grida su un ponte al tramonto o dell'angoscia che il pittore racconta di aver vissuto?

L'iconicità e la somiglianza non possono essere estranee nemmeno alle icone del design, di cui qui ci occupiamo, riportate di recente alla ribalta da Chiara Alessi (2018), oggetti d'uso cui è stato assegnato uno statuto di celebrità: "pezzi cult" e "must have", secondo l'ovvio lessico della pubblicistica. ${ }^{4}$ Anche se il libro è stato scritto per lamentarne la scomparsa - fin dal sottotitolo: $L a$ fine delle icone nel design italiano -, che cosa intende l'autrice con icona nel campo del design e della cultura materiale? Non certo immagini che rappresentano oggetti, quanto oggetti che rappresentano altro, ad esempio la stessa autorappresentazione del prodotto, come Chiara Alessi osserva a proposito dell'iconicità della Moka Bialetti: «Verrebbe da dire che [...] l'iconicità in questo caso risieda nell'iconicizzabilità, ossia nel fatto che nella sua silhouette sia riconoscibile proprio il prodotto che tutti abbiamo in mente quando pensiamo alla moka [...]» (Alessi 2018: 12-13).

Ma cerchiamo innanzitutto di vedere quale potrebbe essere il contributo della semiotica nella definizione di questo particolare ambito dell'immaginario culturale - mettendo per poco da parte i segni visivi, le illusioni referenziali, i divi da copertina e il cestino in fondo ai nostri desktop.

\section{La somiglianza a posteriori}

Se vogliamo tentare di fornire un inquadramento semiotico delle icone del design potremmo dire che nel design è o diventa iconico quel prodotto che presenta almeno i seguenti caratteri generali:

1) in primo luogo, il suo aspetto esteriore e sensoriale (forma, materia, colore, ecc.) si presenta come originale, immediatamente riconoscibile e memorizzabile, marcando una differenza con ogni altro prodotto della stessa classe merceologica che lo ha preceduto;

2) in secondo luogo, tale aspetto estetico è autonomo rispetto a ogni altro valore del prodotto (tecnologico, funzionale, economico, ecc.), che in tal modo, seppure presente e ineliminabile, passa in secondo piano;

3 Ci riferiamo alla nota polemica sull'iconismo fra Umberto Eco e Tomás Maldonado, documentata in Ponzio (1976) e discussa in modo esemplare da Rossella Fabbrichesi Leo (1983).

4 Per una panoramica visuale e documentaria si veda i siti web Vintage Design Trade Show di Amsterdam <https://www.design-icons.com> e Icon Design <https:// icondesign.it/tag/icone-del-design>; online il 31 maggio 2019. 


\section{Ocula}

Vol 21, No 22 (April 2020) • DOI: 10.12977/ocula2020-21

Anna Riboldi e Salvatore Zingale | Oggetti persistenti. La somiglianza a posteriori nelle icone del design

3) in terzo luogo, il prodotto ha la capacità di imporsi all'attenzione pubblica, spesso in tempi lunghi, fino a diventare rappresentativo di un'epoca, di una determinata poetica, di una pratica d'uso;

4) un quarto carattere è la capacità del prodotto di auto-eleggersi a modello o archetipo della tipologia merceologica o mediatica cui appartiene, mostrandosi come motore di innovazione o propagatore di nuovi valori.

Nonostante ciò, piace pensare che la nozione di icona culturale, nel design come in altri casi, abbia pur sempre a che fare con l'icona peirceana, dove ribadiamo - più che imitazione di oggetti, la somiglianza è riconoscimento di qualità e di relazioni possibili da un oggetto all'altro.

Il problema è che cosa va inteso con "oggetto". Vediamo due casi apparentemente simili, di fatto con una interessante differenza. Nel logotipo della compagnia petrolifera Shell l'iconicità è sostenuta dal riferimento all'oggetto "conchiglia"; in quello della multinazionale di abbigliamento sportivo Nike, l'oggetto veicolato può essere individuato sia nel referente stilizzato (l'ala della Nike di Samotracia) sia nei valori di dinamismo, agilità, ambizione e brama di vittoria. E quest'ultimo prevale da tempo sul primo. L'oggetto della rappresentazione, insomma, non è necessariamente un oggetto fisico; e forse, a dire il vero, non lo è quasi mai. Ogni brand infatti tende a imporsi a prescindere dalla figurazione esplicita della sua immagine coordinata.

Questo ci spinge a pensare che l'iconicità nei prodotti del design abbia il suo perno in un altro tipo di "somiglianza", che a prima vista può sembrare paradossale. Proponiamo di chiamarla "somiglianza a posteriori". Si tratta di un'ipotesi di lavoro che qui vogliamo discutere e sperimentare. E l'ipotesi parte dall'osservazione per cui le icone del design raramente sono tali perché assomigliano a qualcosa di già dato, a un contenuto presente nell'enciclopedia o nell'immaginario collettivo; al contrario, in questo caso gli artefatti sono iconici perché assomigliano a qualcosa cui lo stesso artefatto ha dato vita, quasi sempre involontariamente, e di cui prima dell'artefatto nessuno poteva prevedere l'esistenza.

La rassegna minimale che qui presentiamo e le argomentazioni che l'accompagnano ci diranno quanto l'ipotesi possa reggere. Ma un'osservazione di metodo va posta fin da ora. Come si sarà compreso, si tratta di affrancare la nozione di somiglianza - e, con questa, quella di iconicità - dalla funzione meccanicistica in cui sembra essere stata confinata. Perché la somiglianza precede le sue applicazioni alla figurazione pittorica. In altri termini, la somiglianza non sussiste solo come corrispondenza o proiezione punto a punto, analogamente a quanto avviene nelle trasformazioni geometriche, o come nella corrispondenza fra il dipinto di un cavallo e l'animale empirico nel noto esempio disegnato da René Magritte nel 1929 in Les Mots et Les Images. Un rapporto di somiglianza può essere infatti stabilito anche tra un artefatto $\mathrm{e}$ una forma di manifestazione del senso o del sentire comune che proviene dalla storia e dall'esperienza vissuta.

Nei prodotti del design o di altri artefatti materiali e comunicativi la somiglianza va quindi cercata in relazione agli avvenimenti collettivi della loro epoca, più precisamente nei valori che tali avvenimenti hanno fatto emergere; 


\section{Ocula $^{22}$}

Vol 21, No 22 (April 2020) • DOI: 10.12977/ocula2020-21

Anna Riboldi e Salvatore Zingale | Oggetti persistenti. La somiglianza a posteriori nelle icone del design

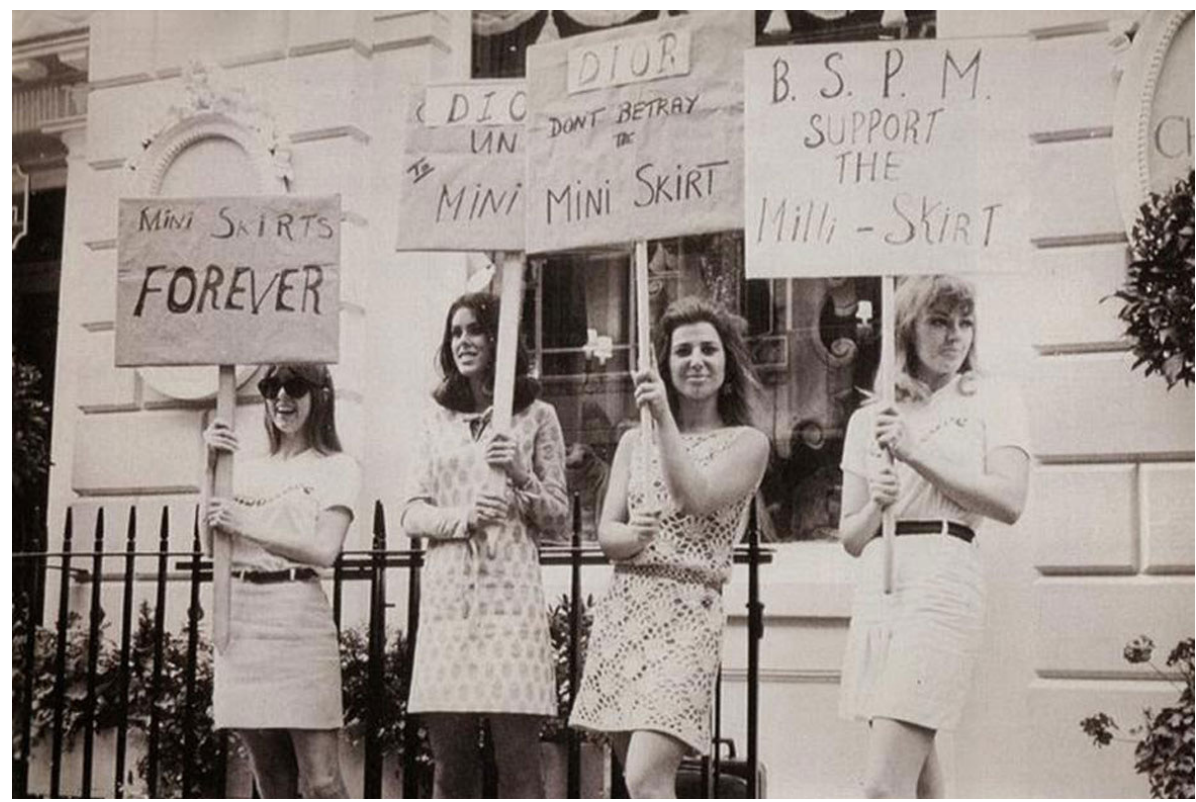

Figura 1. La minigonna di Mary Quant.

come se tali artefatti nel corso della storia avessero assorbito quei valori, fino a diventarne l'immagine più rappresentativa: la loro icona.

Ciò potrebbe costituire il quinto carattere generale che ci permette di definire "iconico" un artefatto o prodotto, che così formuliamo:

5) un prodotto diventa iconico quando mette in atto una somiglianza a posteriori, dove il contenuto referenziale viene posto come esistente solo a seguito della sua affermazione commerciale e culturale, ossia associato a quel prodotto in virtù della sua capacità di emergere e predominare in una certa epoca o in mondi culturali.

L'iconicità della minigonna di Mary Quant (fig. 1), ad esempio, ha come contenuto gli anni della Swinging London - «un'epoca che ha sognato la libertà totale, l'abbattimento di ogni tabù comportamentale» (Renzi in Bonfantini e Renzi 2001: 69) -, anni il cui senso storico viene visualmente significato, fra gli altri, proprio da quell'indumento che ha sovvertito il modo di vestirsi e dato una decisiva spinta alla rivoluzione sessuale.

Ma icona, prima ancora che un termine adottato dalla semiotica, è un termine religioso, che lascia pensare all'inviolabilità dell'immagine. Occorre quindi comprendere se questa connotazione, quando parliamo di "icona", persista o sia del tutto svanita.

\section{Icone del divino e del profano}

Come si diceva, per la storia dell'arte l'icona ha origine nel cristianesimo bizantino ed è una rappresentazione visiva sacra del volto di Cristo, della Madonna o di un santo, realizzata su tavola di legno e impreziosita con dorature (cfr. Kitzinger 1954; Velmans 2000; Bettetini 2006). 


\section{Ocula}

Vol 21, No 22 (April 2020) • DOI: 10.12977/ocula2020-21

Anna Riboldi e Salvatore Zingale | Oggetti persistenti. La somiglianza a posteriori nelle icone del design

Due sono gli attributi che elevano l'icona religiosa a metafora efficace per sondare la natura di ciò che qui chiamiamo icone culturali. In primo luogo, il carattere impersonale e astratto del soggetto raffigurato, come risposta al periodo iconoclastico che contraddistinse l'evoluzione del cristianesimo ortodosso: ciò si esprime attraverso la bidimensionalità della composizione, l'uso allegorico del colore e la scelta del soggetto reiterata tra tipologie stabilite. Nelle icone la figura perde autonomia e corporeità, a scongiurare ogni pericolo di idolatria da parte del suo osservatore e a ricordare che la centralità è affidata, più che all'oggetto della rappresentazione, alla rappresentazione stessa e all'atto artistico in sé: atto peraltro guidato, secondo la teologia ortodossa, proprio dalla volontà di Dio. Il secondo aspetto concerne il rapporto tra queste opere d'arte e i luoghi di culto in cui sono esibite. Le icone nelle chiese dominano l'iconostasi, il "posto delle immagini", quella struttura divisoria che separa gli spazi dedicati ai fedeli dal presbiterio: un luogo semiotico per eccellenza, come una membrana in grado di scindere metaforicamente l'umano dal divino pur consentendo una comunicazione, un passaggio, un guado tra le due dimensioni.

Che si considerino in sé o in funzione degli ambienti che le ospitano, le icone bizantine e russe incarnano la mediazione tra il fedele e il divino, senza sostituirsi a quest'ultimo, bensì divenendone rappresentazione atemporale ed evanescente. Il medesimo ruolo è assunto dalle icone culturali e, nello specifico, dalle icone del design: gli artefatti sono segni e quindi non posseggono i caratteri, le qualità, dell'epoca in cui sono stati prodotti o dell'epoca che li ha resi noti, proprio come l'immagine dipinta della Vergine non è Maria di Nazareth. Gli oggetti iconici non presentano gli stessi attributi del proprio contesto storico o d'uso, ma ne operano una sintesi e ne distillano il carattere. Infine, in qualche modo, la consacrazione degli artefatti a icone è "dettata" dall'epoca in cui questo processo avviene (per ragioni funzionali o stilistiche), esattamente come le icone bizantine sono un messaggio divino che prende forma attraverso la mano del profeta-iconografo.

Diventano icone quegli artefatti di design che condensano nella propria natura il senso e i valori dell'ambiente in cui nascono o del contesto nel quale la loro presenza diventa pervasiva, in termini fisici e di diffusione commerciale oppure in termini di relazione con la società, relazione che non necessariamente deve essere agita tramite il possesso o l'uso, ma che può limitarsi al semplice riconoscimento. Hanno chance di diventare icone tutti gli artefatti che provengono dalle diverse aree del progetto: dal design del prodotto a quello della comunicazione, dall'architettura al progetto di interni, dal car design alla moda e così via.

\section{Artefatto iconico e abiti sociali}

Per esplorare il tema delle icone del design non si può prescindere dalla lettura dell'opera di Chiara Alessi, Le caffettiere dei miei bisnonni (2018). L'autrice è pronipote di Giovanni Alessi e Alfonso Bialetti, fondatori delle omonime aziende e uniti dal comune destino di aver prodotto, in maniere e con 


\section{Ocula ${ }^{22}$}

Vol 21, No 22 (April 2020) • DOI: 10.12977/ocula2020-21

Anna Riboldi e Salvatore Zingale | Oggetti persistenti. La somiglianza a posteriori nelle icone del design

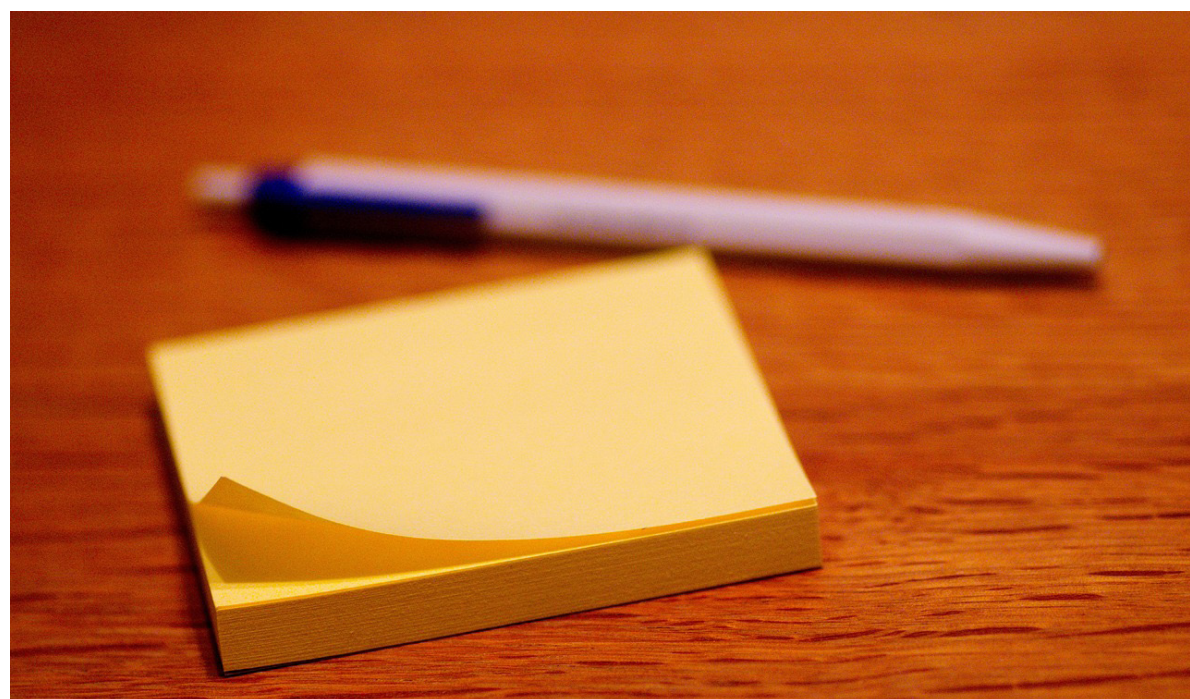

Figura 2. Il Post-it di 3M (1974).

intenzioni diverse, alcune icone dell'italianità per antonomasia. Chiara Alessi individua tre condizioni necessarie, complementari a quelle esposte prima, alla trasformazione di un artefatto in icona del design. Questi attributi non sono necessariamente compresenti o tra loro proporzionati.

Innanzitutto, può diventare icona un artefatto con una funzione, più o meno percepita, che introduce delle alterazioni antropologiche nei gesti, nei rituali, negli abiti del pubblico cui si rivolge. Questo caso è ben rappresentato dai foglietti Post-it (fig. 2), inventati per serendipità nel 1974 da Arthur Fry in risposta alla necessità (del tutto personale) di disporre di segnalibri per l'innario che adoperava quando cantava nel coro in chiesa. ${ }^{5}$ Oltre che per gli effetti sulla società, l'esempio richiamato è singolare per via della storia legata alla genesi di questi foglietti semi-adesivi, che molto racconta sul pensiero inventivo (cfr. Zingale 2012). Infatti, Arthur Fry lavorava alla 3M, nota multinazionale statunitense, dove alcuni anni prima un suo collega, il ricercatore Spencer Silver, stava studiando una formula per realizzare un adesivo che garantisse elevata tenacia. Questi fallì clamorosamente e fu costretto a scartare i risultati del suo lavoro. Arthur Fry ebbe il merito di reinterpretare l'insuccesso progettuale (un collante con esigua potenza) per trasformarlo in una possibile soluzione a un altro problema (l'esigenza di un segnalibro che si attacchi provvisoriamente alla pagina senza rovinarne la carta). Oggi i Post-it permeano la nostra quotidianità e si sono trasformati in uno strumento indispensabile a supporto delle nostre labili memorie: ne sono invasi i libri degli studenti, i monitor negli uffici, i frigoriferi nelle case. Sono entrati a pieno titolo nelle abitudini e nelle consuetudini, tanto che gli attuali sistemi operativi di computer e smartphone offrono applicazioni digitali per annotazioni ispirate alla stessa ritualità d'uso.

5 Sulla serendipità si rimanda a Dri (1994) e a Merton e Barber (2004). 


\section{Ocula}

Vol 21, No 22 (April 2020) • DOI: 10.12977/ocula2020-21

Anna Riboldi e Salvatore Zingale | Oggetti persistenti. La somiglianza a posteriori nelle icone del design

\section{L'icona come carattere}

Determina l'esistenza di un'icona del design anche il valore icastico del suo disegno. Un artefatto, quindi, non solo ha il compito di rispondere a una funzione pratica, ma deve essere portatore di un'impronta visuale robusta, definita e inequivocabile dell'ambiente storico e culturale che lo ha generato. In altre parole, il valore d'uso di un prodotto deve essere accompagnato da un palese valore simbolico e segnico (Baudrillard 1968), ossia anche dalla sua iconicità. E viceversa.

Non ci si addentrerà nell'esplorazione della dialettica tra valore simbolico e valore funzionale, ma in questo contesto è opportuno puntualizzare che non sempre i due coincidono. Tutt'altro: si annoverano icone del design con alta funzionalità e scarsa iconicità (per esempio, i cosiddetti "oggetti anonimi”, come vedremo) e altre caratterizzate da un'iperbole sul valore simbolico a discapito dell'efficacia d'uso (come lo spremiagrumi Juicy Salif di Philippe Starck). In questa dialettica, l'iconicità è la risultante di un bilanciamento quantitativo, più che qualitativo, tra attributi simbolici e valore esperienziale dell'artefatto. Non è importante, in altri termini, la compresenza delle due qualità, bensì l'intensità con cui l'una, l'altra o entrambe esercitano il proprio potere sull'immaginario collettivo. E la prova si rintraccia proprio in quegli oggetti progettati che, pur mancando di peculiari attributi visuali o, in altri casi, di efficacia d'uso, si trasformano comunque in icone del design: qui il valore predominante è tanto vigoroso da compensare quello assente.

Un esempio che racconta come il carattere visivo possa costituire condizione di esistenza dell'icona è rintracciabile nel campo del type design. Tra diversi casi degni di nota, spiccano quelli specifici delle font Futura ed Helvetica. La storia delle due famiglie di caratteri lineari (più conosciuti con la denominazione "bastone") esprime appieno la ricerca di un'identità, di un gusto visivo nel disegno, rispettivamente come rottura o come adeguamento allo stile della loro contemporaneità.

Disegnato da Paul Renner nel 1928 per la fonderia Bauer di Francoforte sul Meno, il Futura si ispirava agli stessi princìpi formali adottati nell'ambito delle sperimentazioni della Bauhaus, ${ }^{6}$ rappresentate nello specifico dall'Universale di Herbert Bayer (1925) e dallo Stencil di Josef Albers (1926). L'obiettivo delle ricerche di Bayer e Albers era la realizzazione di un carattere privo di ornamentazioni e costruito a partire da elementi geometrici essenziali, dunque di facile riproducibilità. Questo proposito sfociò nella produzione di lettere eccessivamente astratte e dallo schema troppo rigido perché potessero essere apportate correzioni ottiche (cfr. Bringhurst 1992 e Hochuli 2015). L’iconicità delle font della Bauhaus cui si ispirò Paul Renner sovrastava quin-

6 Maschile o femminile? Sia per "font" che per "Bauhaus" adottiamo il femminile, con le seguenti ragioni: "la font" perché prima ancora che dall’inglese deriva dal francese fonte ("fusione"), femminile, che indicava l'insieme dei caratteri tipografici in lega metallica realizzati in una singola sessione di fusione; "la Bauhaus" perché il nome completo è "Die Bauhaus-Schule", anche questo femminile. 
Anna Riboldi e Salvatore Zingale | Oggetti persistenti. La somiglianza a posteriori nelle icone del design

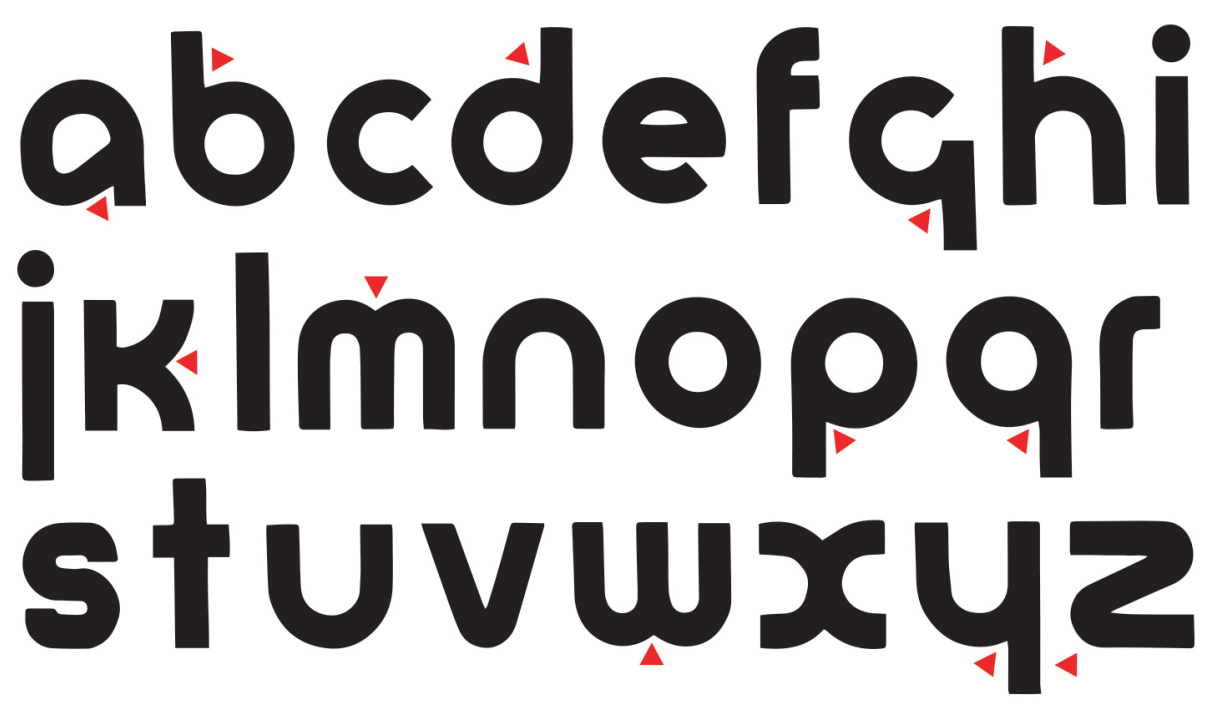

Figura 3. Universale di Herbert Bayer (1925). In rosso si evidenziano le più rilevanti criticità ottiche.

|| |B Y II: I: li|l| I

| R I I III || || | | | I.

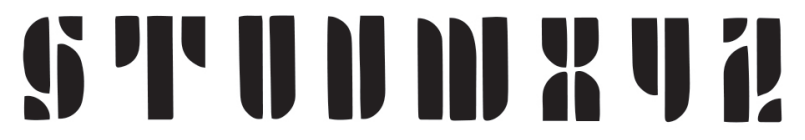

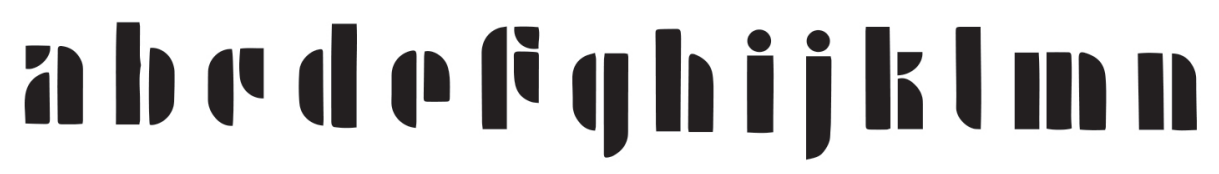

Figura 4. Primo disegno del carattere Stencil di Josef Albers, nelle versioni maiuscola e minuscola (1926).

di la funzionalità, tanto da rendere quegli artefatti tipografici inutilizzabili. Il forte disegno grafico, l'aspetto geometrico, lo stesso principio di "scomporre in fattori primi" la forma delle lettere, e quindi di ottenerne un nuovo disegno attraverso la modulazione di forme geometriche pure (figg. 3 e 4), hanno permesso Bayer e Albers di entrare nella storia della tipografia a pieno titolo. La pressoché completa inutilizzabilità degli stessi alfabeti ha rafforzato il legame iconico tra le loro sagome, il tempo della loro storia e il luogo di provenienza del progetto. 


\section{Ocula}

Vol 21, No 22 (April 2020) • DOI: 10.12977/ocula2020-21

Anna Riboldi e Salvatore Zingale | Oggetti persistenti. La somiglianza a posteriori nelle icone del design

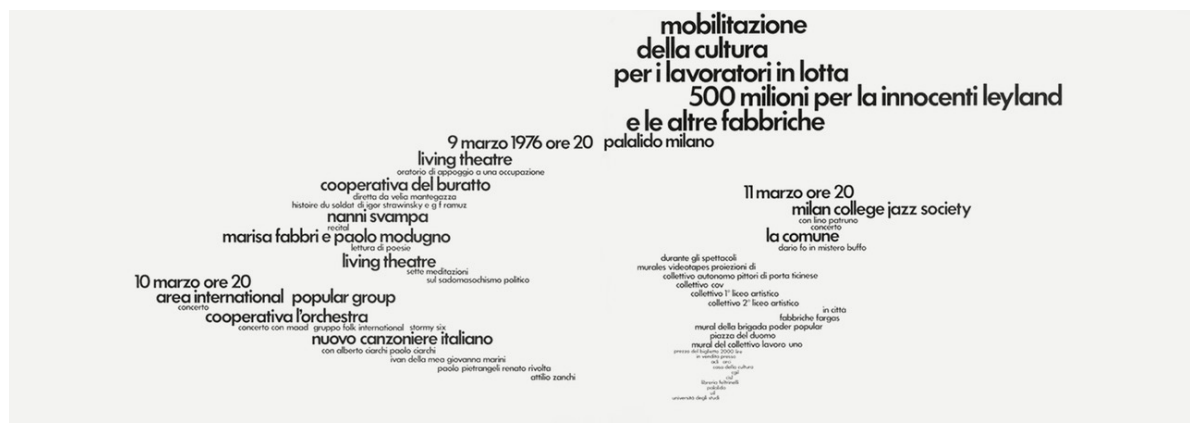

Figura 5. Il carattere Futura impiegato da AG Fronzoni in un suo noto affiche.

Diverso il destino del Futura. Nella sua prima versione, molto aderente ai canoni della Bauhaus, i glifi erano costruiti sulla base di tre figure geometriche pure: quadrato, cerchio e triangolo. Per questa ragione, l'eccessivo rigore compositivo limitava il disegno delle lettere che, specie nel minuscolo, assumevano configurazioni inconsuete, dunque poco leggibili. A differenza dei suoi predecessori, Renner riuscì ad apportare delle variazioni ottiche al suo alfabeto: assottigliò i tratti orizzontali, rifinì i punti di raccordo per renderli più snelli, infine bilanciò le altezze delle lettere con un lieve incremento nei glifi su base tonda e triangolare (per esempio, "o", "c", "v") rispetto a quelli su base quadrata (come "z", "L", "T"). Si può interpretare la scelta di rompere gli schemi e di accettare il compromesso con i vincoli di leggibilità come una doppia rottura del Futura rispetto al proprio tempo: da un lato, l'adesione al movimento del geometrico sottende l'intenzione di restituire razionalità a una tipografia ancora reduce dei tratti sinuosi tipici dell'Art Nouveau, per adattarla al nuovo gusto progettuale; dall'altro, il superamento dei vincoli della Bauhaus rappresenta l'adesione al compromesso tra valore iconico e valore d'uso insito nella buona sorte di questo progetto.

Per quando spesso sconsigliato in alcuni ambiti, per via della scarsa leggibilità del minuscolo, il Futura ha trovato diversi interpreti che lo hanno elevato a icona grafica. In particolare, ci piace ricordare la sua trasformazione in poesia concreta o visiva da parte di AG Fronzoni che, così come con l'Helvetica, ne ha esaltato il rigore formale (fig. 5).

L'Helvetica, disegnato da Max Miedinger nel 1957 per la fonderia svizzera Haas, a sua volta si ispirava alla struttura formale dell'Akzidenz Grotesk e fu inizialmente concepito per modificarne le proporzioni tra maiuscolo e minuscolo e per revisionarne alcuni dettagli. Questi aggiustamenti diedero luce a un alfabeto autonomo, dalla forma essenziale, dai pieni ben marcati e dall'estrema adattabilità, per merito delle sue trentaquattro varianti di tono, larghezza e inclinazione. La sua compostezza ed eleganza formale, unite al disegno razionale, lo resero il carattere tipografico dominante nella grafica degli anni Sessanta, e, insieme al suo antenato Akzidenz Grotesk, uno dei pochi alfabeti accettati nello stile grafico della scuola svizzera (cfr. Hochuli 2015).

Futura ed Helvetica costituiscono la risposta progettuale all'esigenza di disporre di lettere chiare e impersonali, con un'aura seria e rigorosa, alleg- 


\section{Ocula ${ }^{22}$}

Vol 21, No 22 (April 2020) • DOI: 10.12977/ocula2020-21

Anna Riboldi e Salvatore Zingale | Oggetti persistenti. La somiglianza a posteriori nelle icone del design

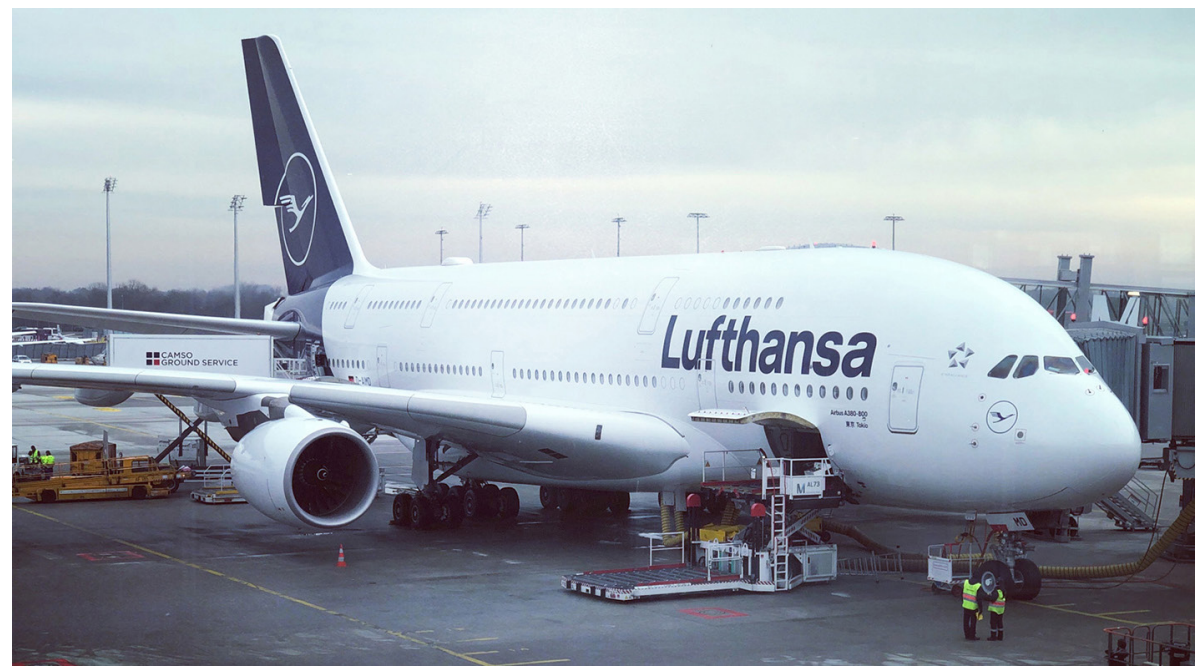

Figura 6. Il carattere Helvetica applicato per il logotipo della Lufthansa.

gerite della classicità graziata. Tutt'oggi in diversi campi del design della comunicazione - editoria, immagine coordinata, segnaletica, ecc. - queste font vengono preferite alle più recenti, proprio per quei valori che così efficacemente riescono a custodire e a comunicare attraverso la peculiarità del loro disegno e delle loro proporzioni (fig. 6).

C'è poi l'Helvetica corretto e reinventato da Bob Noorda per la segnaletica della Metropolitana Milanese (fig. 7), una delle più rilevanti icone grafiche. Il grafico olandese disegnò un carattere ad hoc, chiamato Noorda, adeguando le forme dell'Helvetica all'uso in negativo (bianco su rosso) per evitare l'effetto di amplificazione ottica che diminuiva la leggibilità del carattere di partenza.

Si rende legittimo affermare, quindi e infine, che Futura ed Helvetica sono modelli di icone grafiche atemporali. $\mathrm{O}$, meglio, di icone concettuali.
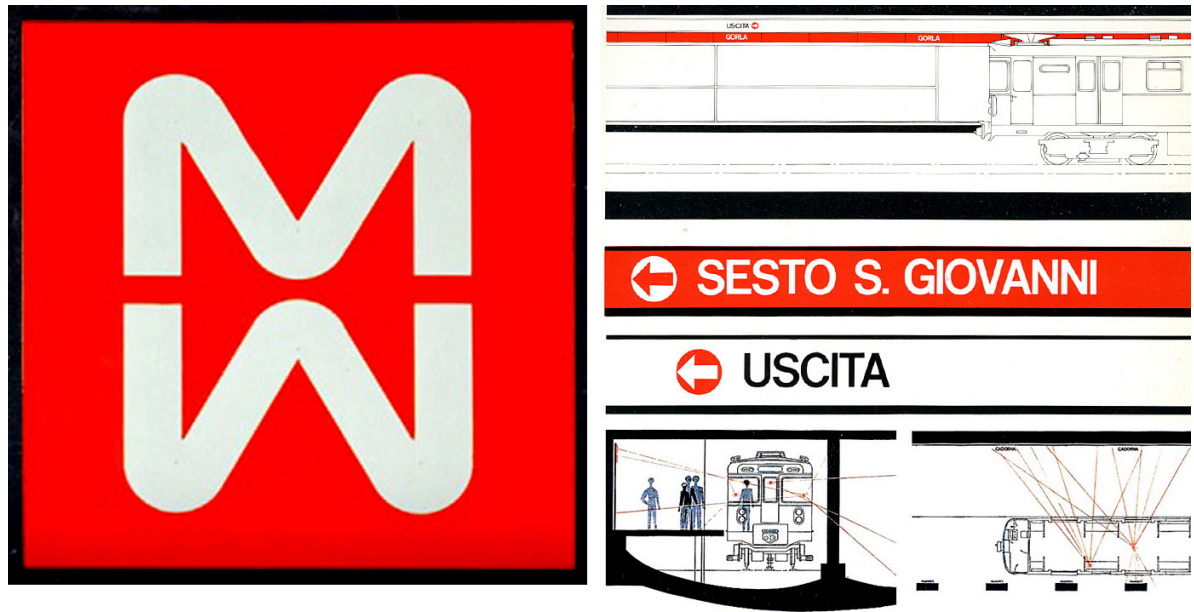

Figura 7. L'Helvetica modificato da Bob Noorda (e rinominato "Noorda") per la segnaletica della Metropolitana Milanese nel 1962. 


\section{Ocula ${ }^{22}$}

Vol 21, No 22 (April 2020) • DOI: 10.12977/ocula2020-21

Anna Riboldi e Salvatore Zingale | Oggetti persistenti. La somiglianza a posteriori nelle icone del design

\section{I canali alternativi dell'icona}

Un'ulteriore proprietà: un artefatto può diventare iconico se entra in contatto con l'immaginario collettivo attraverso canali non consueti, e più precisamente quando è menzionato e diffuso a livello mediatico. Ciò avviene generalmente per mezzo del cinema, dei format televisivi, del web e della letteratura, fino a coinvolgere persino la produzione musicale. Il rimando da parte di un autore rappresenta la conferma del valore culturale dell'artefatto e ha le sembianze di un suggerimento: è opportuno, doveroso, che l'oggetto menzionato sia conosciuto, riconosciuto e ricordato. Il modello della citazione segue il principio dell'ipse dixit, dove l'autorevolezza di un terzo soggetto non viene discussa o messa in dubbio, perché ritenuta affidabile, autentica e al di sopra delle parti. All'aumentare del numero di menzioni, soprattutto quando queste provengono da canali e autori differenti, l'effetto si amplifica. $\mathrm{Ne}$ consegue che, quando il medium fa l'icona, si accresce anche il rischio di accettare il ruolo iconico di un artefatto al solo scopo di evitare un sentimento di distanza dall'immaginario collettivo percepito e, per proiezione, di esclusione sociale.

Uno dei prodotti più citati del design industriale italiano è la Vespa Piaggio (fig. 8), il cui brevetto (datato 23 aprile 1946) appartiene all'ingegnere aeronautico Corradino D'Ascanio, anche noto per essere l'inventore dell'elicottero. Nell'Italia del Dopoguerra, alle prese con la ricostruzione, questo scooter dall'indiscutibile bellezza e dalle qualità tecniche innovative non faticò a diventare metafora di una società in movimento. Un movimento snello, agile, frenetico: «scooter deriva dal verbo to scoot, filare o scivolar via, correre in fretta» (Bonfantini e Renzi 2001: 49-50).

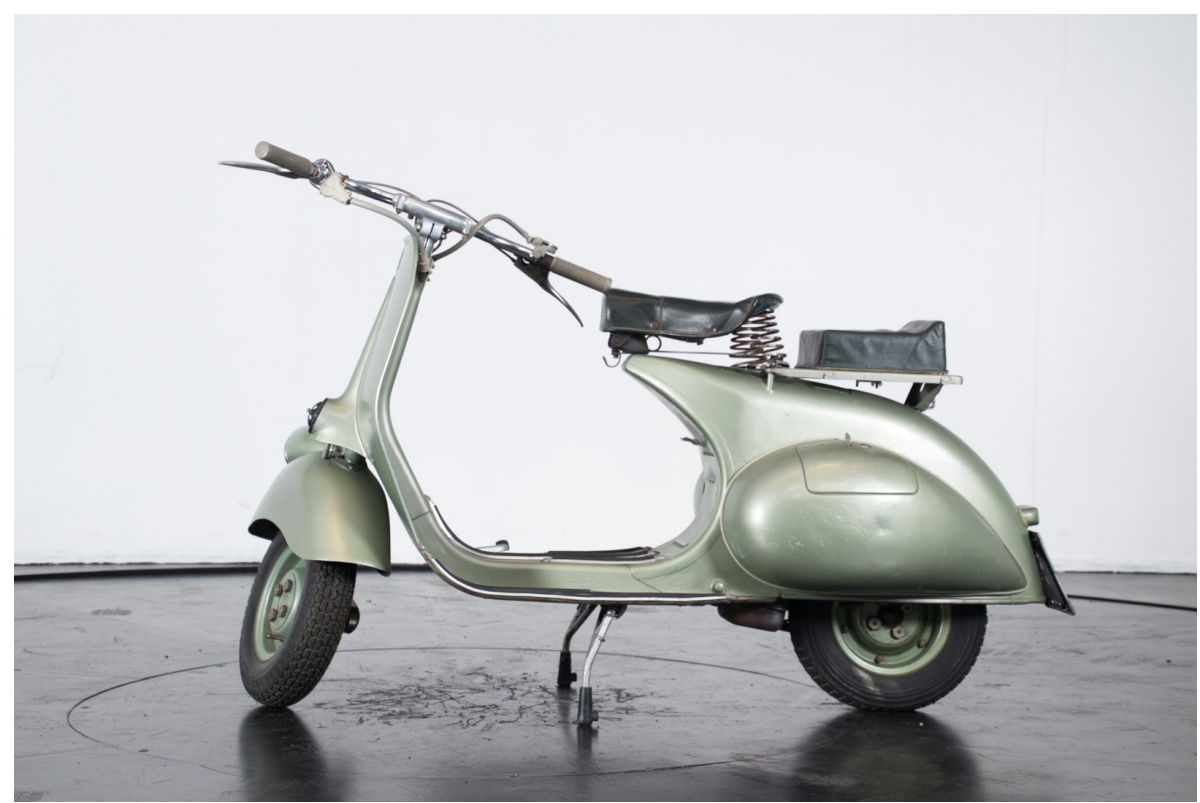

Figura 8. La Vespa Piaggio utilizzata nel film "Vacanze romane” (1953). 


\section{Ocula ${ }^{22}$}

Vol 21, No 22 (April 2020) • DOI: 10.12977/ocula2020-21

Anna Riboldi e Salvatore Zingale | Oggetti persistenti. La somiglianza a posteriori nelle icone del design

Se nello scenario nazionale l'iconicità della Vespa ha principalmente l'effetto di evocare l'umore di uno specifico periodo storico, sul piano internazionale la stessa acquisisce una portata simbolica più imponente, orientata ad altri contenuti: la Vespa diventa icona per eccellenza dell'italianità. Italianità che è già espressa dalla qualità del progetto e della sua produzione industriale, ma che raggiunge il suo culmine grazie all'immagine costruita mediaticamente attorno all'artefatto-icona: un immaginario collettivo a tutti gli effetti artificiale e non sempre verosimile, che attinge da un ricco serbatoio cinematografico: la Vespa Piaggio compare in film come Vacanze Romane (1953), I soliti ignoti (1958), Scarface (1983), 7 chili in 7 giorni (1986), Good Morning, Vietnam! (1987), Caro diario (1993) e altri ancora. L'elenco di tutte le sue comparse sarebbe troppo lungo per essere riportato integralmente. Anche grazie all'ampiezza della risonanza conquistata, alimentata nell'arco di mezzo secolo di menzioni, la Vespa si eleva a quell'icona rappresentativa della cultura italiana il cui ruolo è indiscusso e riconosciuto in tutto il mondo.

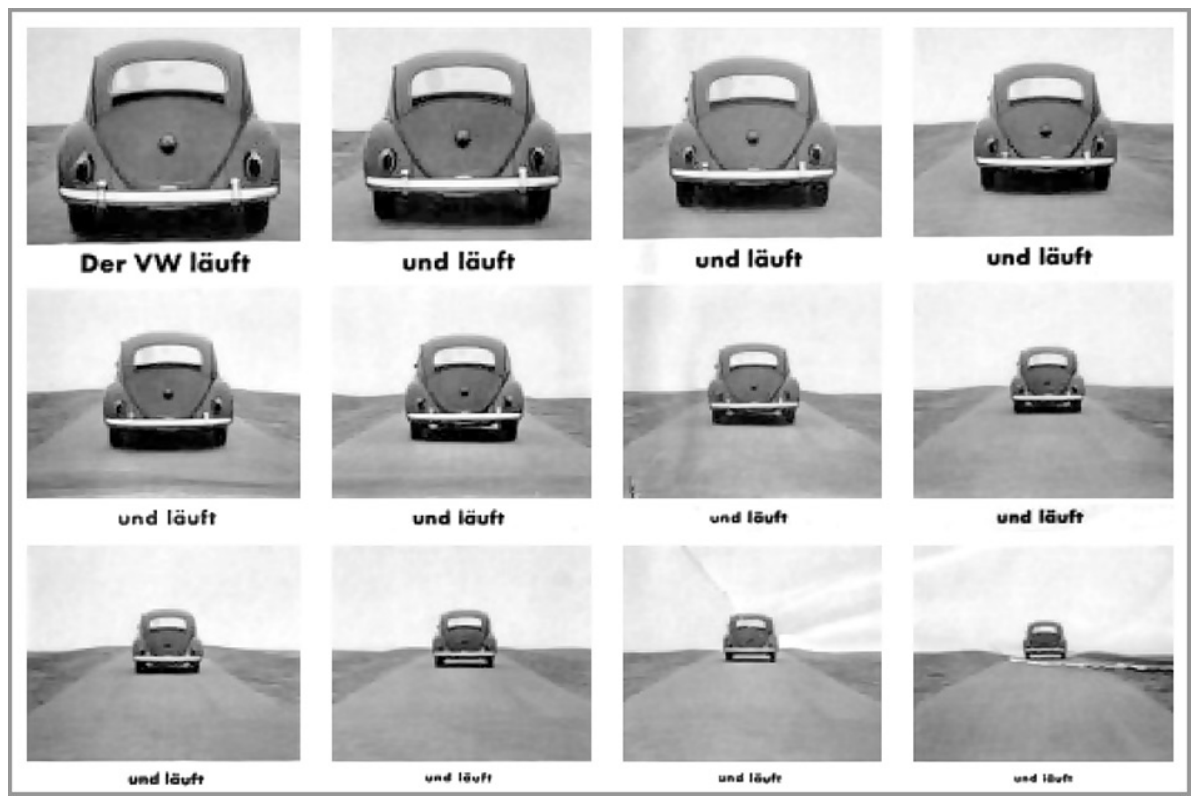

Figura 9. Pubblicità della Volkswagen all'inizio degli anni Sessanta, con headline di Werner Butter.

Analogo il destino di due sorelle maggiori della Vespa, due utilitarie che, in anni recenti, hanno beneficiato dell'onore della citazione e quindi di una seconda vita: la Fiat 500 e il Käfer Volkswagen (il Maggiolino). ${ }^{7}$

L'idea del Maggiolino Volkswagen, come sappiamo, era nata in epoca hitleriana, ma le vicende belliche impedirono che diventasse l'automobile simbolo di quegli anni plumbei, per trasformarsi invece in icona della rinascita: dal-

7 Sull'automobile come uno degli oggetti del Novecento si rimanda a Bonfantini e Renzi (2001). 


\section{Dio è unt esserperfettissimo come und VOLKSWAGEN}

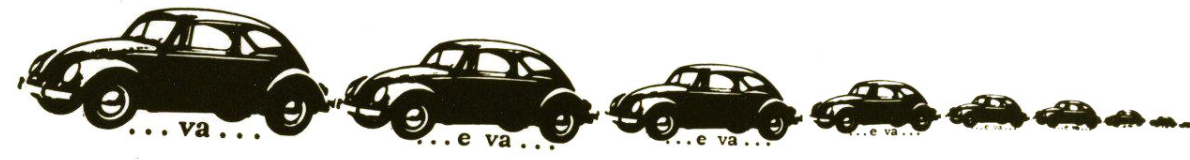

Figura 10. Emilio Isgrò, Come una Volkswagen (1964).

la ricostruzione degli anni Cinquanta fino al Sessantotto e agli anni Settanta. Nel 1964, analogamente a una pubblicità tedesca con un leggendario headline di Werner Butter, il poeta visivo Emilio Isgrò la elevò a icona della perfezione divina, perché è un'auto che va, e va, e va, e va ... (figg. 9 e 10).

\section{Icona, tempo e spazio}

Tutti gli artefatti, iconici o meno, si confrontano e dialogano con una realtà innanzitutto dimensionale, costituita di una spazialità e di una temporalità. Gli oggetti del design (anche visivi) entrano negli ambienti fisici, li permeano, intessono relazioni con gli altri oggetti e interagiscono con l'utente attraverso le qualità esperienziali (funzionali ed estetiche) di cui sono portatori. Inoltre, fanno tutto ciò in un determinato tempo storico, che ha caratteristiche proprie e non trascurabili, sul piano politico, sociale e culturale.

La risposta che scaturisce dalla dialettica tra artefatto e realtà può intraprendere tre sentieri principali. In primo luogo, nella direzione della neutralità: un itinerario poco interessante ma da non trascurare, trattandosi dell'unica via sicura affinché un artefatto non diventi icona. Il secondo percorso è nel verso dell'adesione: l'artefatto è in grado di cogliere i tratti pertinenti dell'ambiente che lo circonda, di interpretarne l'essenza e di trasformarsi in uno dei simboli che lo rappresentano, fino a instaurare un rapporto di identità reciproca con il contesto. La terza strada, come ricorda Chiara Alessi (2018: 32-33), è orientata a un atteggiamento di rifiuto della realtà da parte dell'artefatto, che manifesta uno scarto, una rottura degli schemi, rispetto all'hic et nunc, e che quindi determina una forma di innovazione culturale. Maggiore è la portata di questo distacco, più intenso il valore iconico assunto dall'artefatto.

Un caso che descrive con chiarezza il legame dell'artefatto con il proprio tempo, inizialmente attraverso uno scarto, poi nell'identificazione, è il mo- 


\section{Ocula $^{22}$}

Vol 21, No 22 (April 2020) • DOI: 10.12977/ocula2020-21

Anna Riboldi e Salvatore Zingale | Oggetti persistenti. La somiglianza a posteriori nelle icone del design

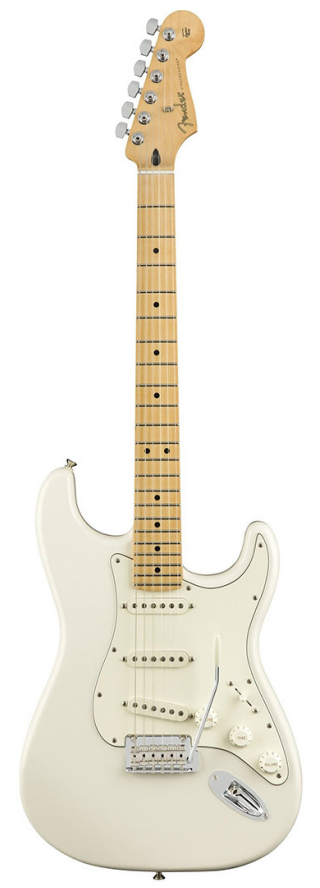

Figura 11. Chitarra Fender Stratocaster.

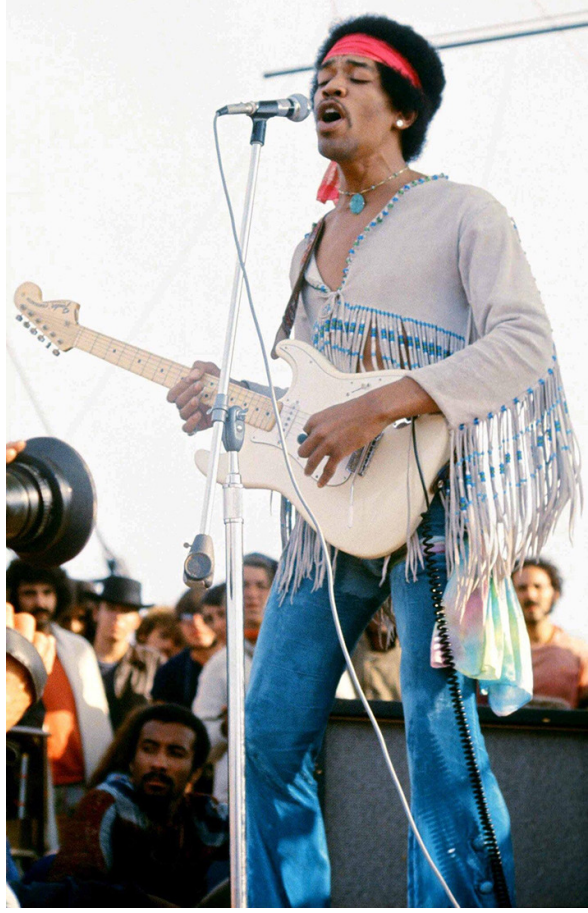

Figura 12. Jimi Hendrix a Woodstock nel 1969.

dello Stratocaster della chitarra elettrica Fender (figg. 11 e 12), perfezionato dal liutaio Leo Fender nel 1954. L'innovazione tecnica legata al disegno di questa chitarra è prima di tutto di tipo funzionale: rispetto ai suoi simili, lo strumento vanta l'aggiunta della tipica "leva del vibrato", con la quale è possibile modulare il suono per aumentare il cosiddetto sustain (la persistenza della nota nel tempo), e conta la presenza di un numero maggiore di pickup, $\mathrm{i}$ trasduttori deputati alla conversione del suono in segnale elettrico. Infine, le modifiche apportate alla cassa armonica, con la tipica rientranza della spalla, inizialmente costituiscono la risposta all'esigenza di raggiungere sulla tastiera uno spettro più ampio di note acute e, secondariamente, definiscono una sagoma che rompe gli schemi rispetto al modello mentale dell'artefatto-chitarra universalmente accettato fino a quel momento.

Il timbro squillante e l'originalità degli effetti sonori definirono una sonorità innovativa e ampliarono le possibilità espressive, distinguendo marcatamente la Stratocaster dai modelli analoghi, per esempio da quelli firmati Gibson. La chitarra Fender cominciò subito a essere privilegiata da un nutrito numero di chitarristi, tra i quali si annoverano George Harrison e John Lennon (Beatles), Eric Clapton, Stevie Ray Vaughan, Frank Zappa, David Gilmour (Pink Floyd), Ritchie Blackmore (Deep Purple) e così via. Anche questa volta, l'elenco è troppo ampio perché sia riportato nella sua completezza.

Ma l'incoronamento della Stratocaster a icona culturale avvenne per mano del chitarrista per antonomasia: Jimi Hendrix. Hendrix si distinse per aver saputo interpretare un sound completamente innovativo, dove la ritmica del 


\section{Ocula}

Vol 21, No 22 (April 2020) • DOI: 10.12977/ocula2020-21

Anna Riboldi e Salvatore Zingale | Oggetti persistenti. La somiglianza a posteriori nelle icone del design

blues e del soul si combinava alle sonorità rock e psichedeliche. Il giovane musicista intrecciò un rapporto quasi ludico con il suo strumento: la stessa abitudine di suonare la chitarra rovesciandola, gesto che derivava dalla sua condizione di mancino costretto a suonare strumenti per destrimani, assunse una connotazione altamente iconica.

C'è, però, dell'altro: la Stratocaster non è un'icona solo perché Hendrix lo è a sua volta. In questo caso, l'ingrediente segreto, la variabile indispensabile e sottesa, è l'epoca storica di cui le due future icone diedero origine a questa alchimia: un tempo costituito di eventi come la guerra del Vietnam e la nascita, per reazione, di un movimento di contestazione e protesta popolare (i cosiddetti hippy); un tempo contraddistinto, parallelamente, dalle battaglie per la conquista dei diritti civili degli afroamericani, sia nel verso dell'integrazione con la società bianca (grazie all'operato di Martin Luther King) sia nell'ottica della contrapposizione (con le Pantere Nere, guidate da Malcolm X). Un tempo che, infine, ospitò Woodstock, il più grande festival della scena rock e psichedelica, durato tre giorni, dal 15 al 18 agosto 1969. Jimi Hendrix, già conosciuto per il suo esordio del 1967 al festival di Monterey, in cui concluse la performance con l'incendio del proprio strumento, a Woodstock era l'ultimo artista previsto dalla scaletta (fig. 12). Durante la sua esibizione interpretò con la sua Stratocaster una versione dell'inno americano completamente distorta e stonata, come atto di pacifica protesta contro le scelte belliche degli Stati Uniti. Questa immagine suggestiva, pregna di significati e di associazioni, è rimasta nell'immaginario collettivo e ha attraversato la storia con una risolutezza non facilmente contrastabile.

Come si è intuito relativamente al caso Stratocaster, sul piano del tempo storico si rileva che le condizioni più propizie all'evoluzione di un prodotto del design in icona appartengono a quelle epoche in cui il succedersi degli eventi è ordinato e contraddistinto da un inizio, uno sviluppo e una fine. Tre sono le spiegazioni. Primo: perché il valore iconico dell'evento entra in relazione con quello dell'artefatto, con cui tesse una rete di associazioni. Secondo: perché gli avvenimenti di un certo rilievo, con le loro influenze e i loro strascichi sulla storia, segmentano il tempo in unità circoscritte, descrivibili attraverso poche qualità salienti. Infine: perché questi stessi attributi richiedono un intervallo sufficiente per permeare tutti gli aspetti della realtà ed essere metabolizzati.

Uno dei problemi della nostra contemporaneità, ricca erede di icone del design e al contempo sterile di novità, si annida proprio nella natura del tempo attuale. La tecnologia si aggiorna a ritmi iperbolici e le aziende affollano il mercato di prodotti con l'intenzione di ottenere un vigoroso impatto sul presente, senza interessarsi del loro incerto futuro. Tutt'altro: la cosiddetta "obsolescenza programmata”, in quest'ottica, costituisce un effetto coerente con l'esigenza di adattare il passo del consumo a questa nuova frenesia ideativa e produttiva.

In più, il web ha generato un altro tempo: i fili invisibili di questa rete, in cui circolano informazione e conoscenza, connettono paesi e culture del mondo tra loro anche molto lontane. L'accessibilità ai contenuti in tempo reale, la possibilità di un costante aggiornamento sui fatti, lo stesso entrare in contatto 


\section{Ocula $^{22}$}

Vol 21, No 22 (April 2020) • DOI: 10.12977/ocula2020-21

Anna Riboldi e Salvatore Zingale | Oggetti persistenti. La somiglianza a posteriori nelle icone del design

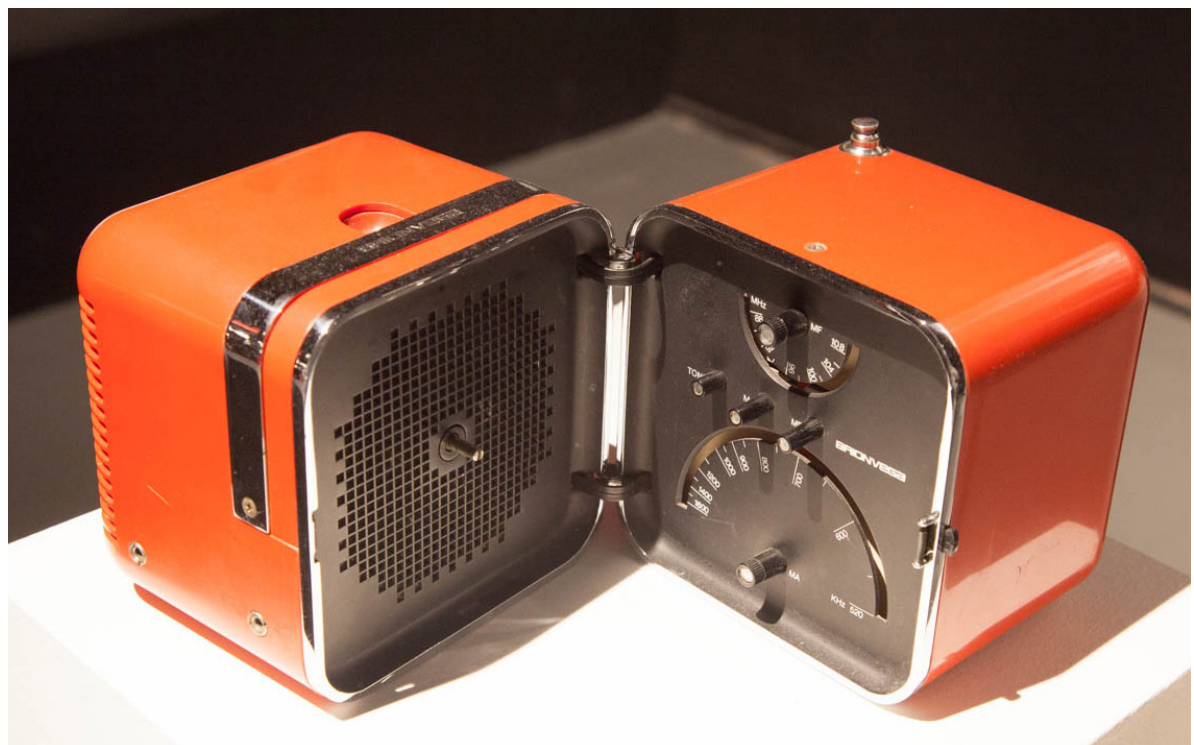

Figura 13. La radio “TS502” di Marco Zanuso e Richard Sapper per Brionvega.

con tecnologie e abiti inesplorati abbatte ogni limite dimensionale e, di conseguenza, rende la società e la storia delle entità non definite, liquide (cfr. Bauman 2000 e Manzini 2018). Il tempo di oggi è così frammentato che, per usare una metafora matematica, sembra evidente il passaggio dal discreto (scandito da una concatenazione di eventi) al continuo (dove la durata delle epoche è tanto ridotta da renderle indistinguibili). Un tempo senza più epoche, che al massimo genera stagioni, più che vantare icone può ambire a proporre tormentoni destinati a un imminente oblio.

La dialettica tra oggetti iconici del design e tempo non concerne esclusivamente la categoria della contemporaneità. Come si è già visto nel caso della tipografia della Bauhaus, i destini degli artefatti iconici possono essere tra loro molto diversi, in relazione al mantenimento della propria funzione primaria o alla sua conversione in funzioni ulteriori, reinterpretate e in grado di costituire un collante con nuovi tempi e nuovi spazi.

Le icone che rievocano precisi momenti storici, in forte relazione con il proprio tempo (o con il tempo che le ha consacrate in quanto tali), incontrano due possibili sviluppi, che dipendono dalla loro capacità di generare funzioni secondarie una volta persa (o indebolita) quella primaria. In presenza di una reinterpretazione e dell'attribuzione di nuovi valori, l'oggetto mantiene il ruolo iconico rispetto alla sua epoca e, insieme, crea relazioni con il tempo attuale. È il caso, per esempio, della radio TS502, progettata da Marco Zanuso e Richard Sapper per Brionvega nel 1962: alla funzione per cui il dispositivo è stato originariamente ideato subentra una più consistente valenza iconica, per la quale l'artefatto, anche quando perfettamente adoperabile, assume la veste di opera d'arte, di scultura del design, di oggetto da collezione (fig. 13). Se invece la funzione primaria non è compensata, il destino dell'icona è di dissolversi in qualche soffitta, per comparire in sporadici discorsi nostalgici. 


\section{Ocula ${ }^{22}$}

Vol 21, No 22 (April 2020) • DOI: 10.12977/ocula2020-21

Anna Riboldi e Salvatore Zingale | Oggetti persistenti. La somiglianza a posteriori nelle icone del design

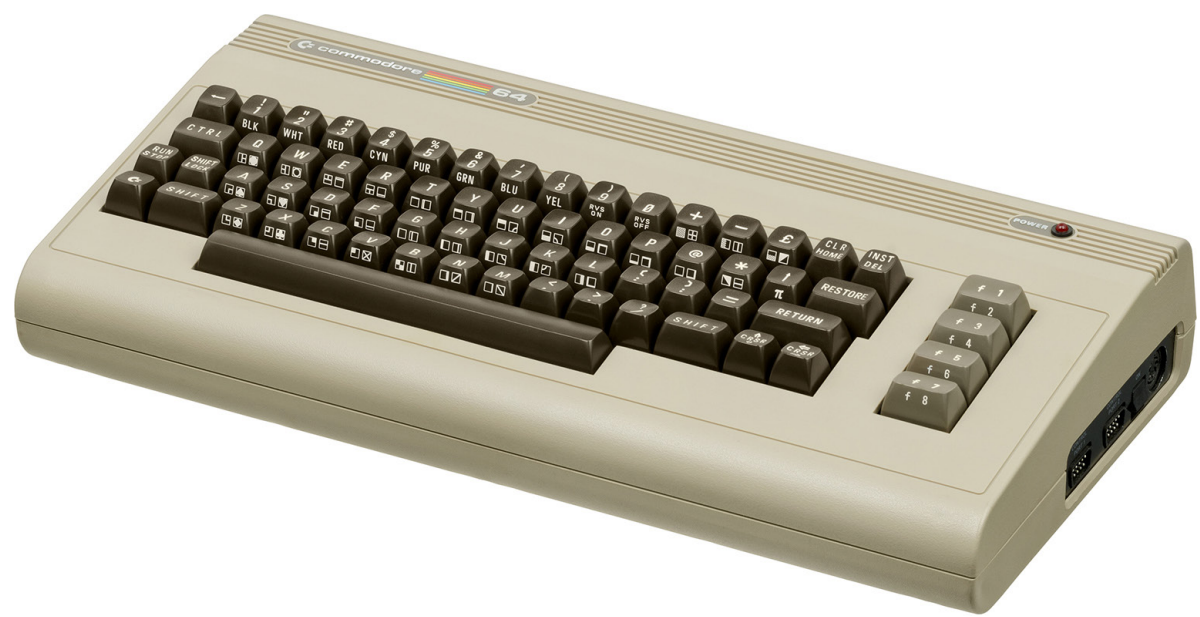

Figura 14. La tastiera del Commodore 64 di Microsoft.

Un esempio è rappresentato dall'home computer Commodore 64, prodotto da Microsoft nel 1982 (fig. 14). Il suo indubbio valore iconico, dovuto al salto innovativo provocato e all'incredibile successo in termini di vendite, oggi non può essere sufficientemente esaltato, poiché la funzione primaria (di natura informatica) non è supportata da altre qualità, per esempio estetiche.

Qual è, però, il futuro dell'icona quando la sua funzione primaria non decade e, al contrario, si mantiene vigorosa? Come si è già mostrato nell'analisi dei casi tipografici del Futura e dell'Helvetica, esistono artefatti che, per via del consistente livello funzionale raggiunto, attraversano le epoche e conservano il proprio ruolo indipendentemente dallo scorrere del tempo. La loro iconicità, quindi, si fonda su altri valori, che non appartengono alla storia.

Un esempio che aderisce a questa eventualità si può osservare nel mondo dell'editoria e coinvolge il "formato tascabile" tipico delle edizioni economiche (fig. 15). Le origini del libro tascabile, per la precisione, precedono l'invenzione della stampa e sono legate alla produzione di piccoli volumi chiamati "libri da bisaccia", realizzati su supporto cartaceo da scribi non professionisti. Nonostante la vocazione popolare di questi artefatti, la loro diffusione non fu mai significativa a causa dell'elevato costo di produzione. Cinquant'anni dopo l'avvento della stampa a caratteri mobili, però, il tipografo Aldo Manuzio si adoperò per conferire dignità e prestigio alle pubblicazioni in formato ridotto che, questa volta, risultavano economiche per via dell'abbattimento dei costi conseguente alla produzione seriale. L'intento di Manuzio si concretizzò con l'edizione aldina delle Bucoliche di Virgilio, pubblicata nel 1501, e in seguito si sviluppò nella creazione della prima collana di classici. L'opera del tipografo ed editore contribuì a quel lento processo di desacralizzazione del libro che trovò, in seguito, massima espressione nel corso del Novecento.

Il formato tascabile ha quindi attraversato più di cinque secoli di storia e, per come è conosciuto oggi, veicola due significati fondamentali. Il primo, puramente economico, concerne la garanzia (spesso illusoria) di un prezzo più vantaggioso. Il secondo, di natura culturale e paratestuale, è la certezza 


\section{Ocula $^{22}$}

Vol 21, No 22 (April 2020) • DOI: 10.12977/ocula2020-21

Anna Riboldi e Salvatore Zingale | Oggetti persistenti. La somiglianza a posteriori nelle icone del design

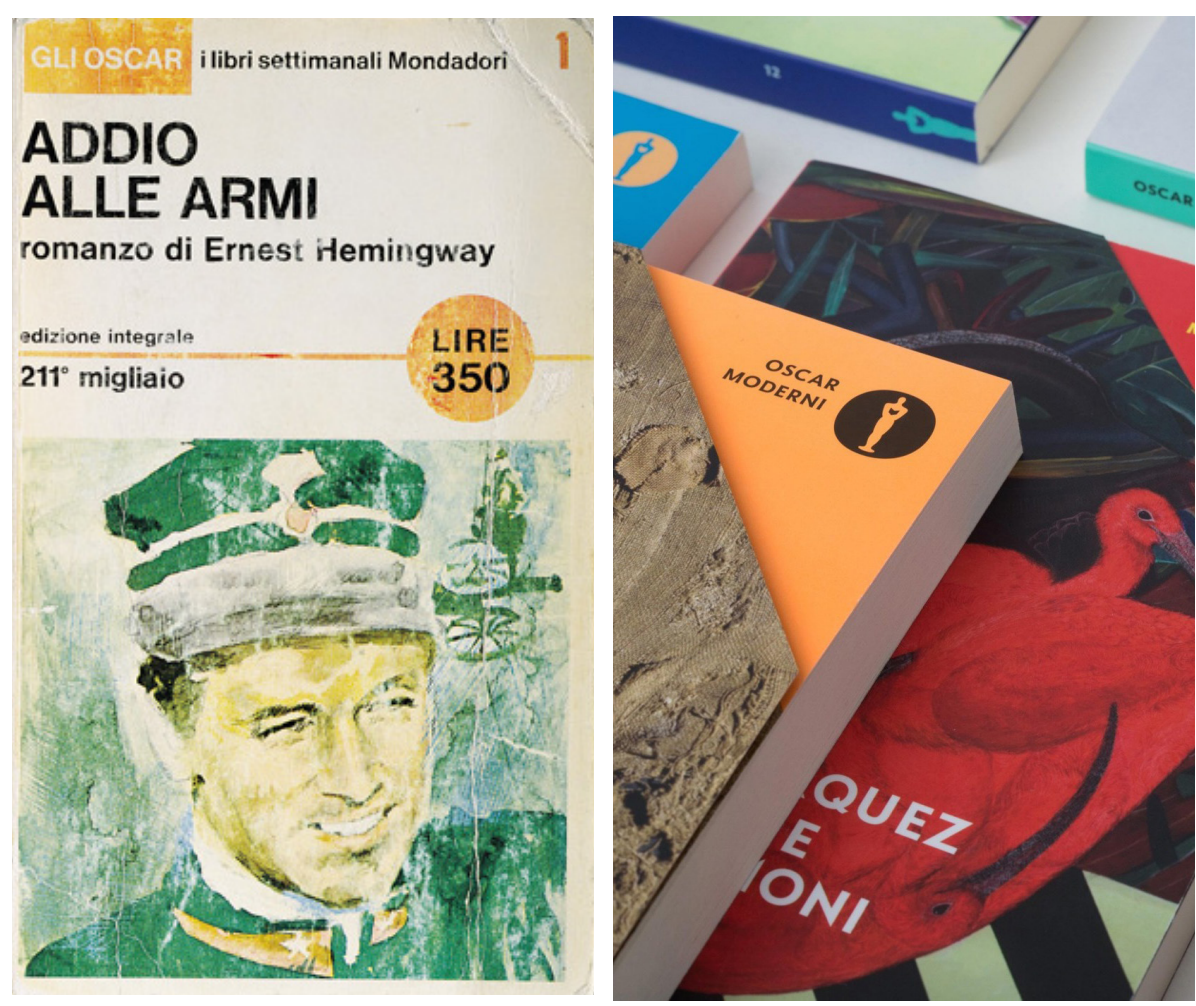

Figura 15. Gli Oscar Mondadori. A sinistra la copertina del primo volume pubblicato nel 1965.

di una selezione che si fonda sul concetto di riedizione: l'edizione tascabile rappresenta la consacrazione del titolo da parte dell'editore, della critica e del pubblico (cfr. Genette 1987).

L'iconicità del tascabile, quindi, non concerne i momenti storici legati alla sua invenzione e reinvenzione: questo formato è riconosciuto dall'immaginario collettivo per qualità che non restituiscono il senso della sua epoca e che, piuttosto, si riferiscono al senso dell'edizione e dell'opera. Tutti riconoscono un tascabile, in pochi conoscono le sue origini. Ciò dimostra che icona e tempo non sempre si accompagnano. Anzi, a volte non si incontrano proprio.

\section{Icone del design e autore collettivo}

In un discorso sulle icone del design, il tema autoriale sarebbe trascurabile, collaterale: così come è possibile individuare l'autore di un "oggetto-di-design", non sempre si è in grado di indicare la paternità di un "oggetto-icona". Questo perché le icone non nascono in seguito a una presa di posizione, a un atto decisionale e intenzionale, ma si sviluppano in un processo autonomo, incontrollato e legato a più variabili interrelate (la tecnica, il contesto, l'immaginario collettivo e così via). L'autore, se c'è, è l'ideatore dell'artefatto. È tutto il resto a generare l'icona.

Ne deriva una prima considerazione: non è possibile manovrare l'attività progettuale allo scopo di concepire a priori degli artefatti destinati a trasfor- 


\section{Ocula ${ }^{22}$}

Vol 21, No 22 (April 2020) • DOI: 10.12977/ocula2020-21

Anna Riboldi e Salvatore Zingale | Oggetti persistenti. La somiglianza a posteriori nelle icone del design

marsi in icone, proprio perché i fattori che concorrono alla metamorfosi non sono prevedibili.

La figura dell'autore, però, se esaminata nei suoi diversi gradi di esistenza, costituisce una buona occasione per indagare su altri aspetti che concernono le icone e la loro genesi.

Il grado zero dell'autorialità è quello dell'autore implicito. Si riscontra nei cosiddetti “oggetti anonimi”, come quelli che Bruno Munari, nel 1972, candidava per un ipotetico Compasso d'Oro a ignoti. In Da cosa nasce cosa (Munari 1981) si trova un esauriente elenco, dalla sedia sdraio alla lampada da garage, dalla spilla da balia alla molletta per il bucato (fig. 16). In generale, l'origine di questi artefatti è indefinita nel tempo e nello spazio. Gli oggetti anonimi rappresentano il risultato di una progettazione comune, avvenuta per via di una stratificazione di modifiche, deviazioni e interpretazioni di artefatti precedenti, al fine di adattarli a necessità più complesse e in risposta alle carenze dell'offerta industriale (cfr. Branzi 2007). Gli oggetti anonimi nascono da un sapere e da un'intelligenza popolare, da un'inventiva collettiva. Ciò che consacra questi artefatti a icone del design è la funzionalità, più che il tratto estetico, proprio perché «questo tipo di produzione spontanea [è] frutto della necessità e non del consumismo» (Branzi 2007: 49).

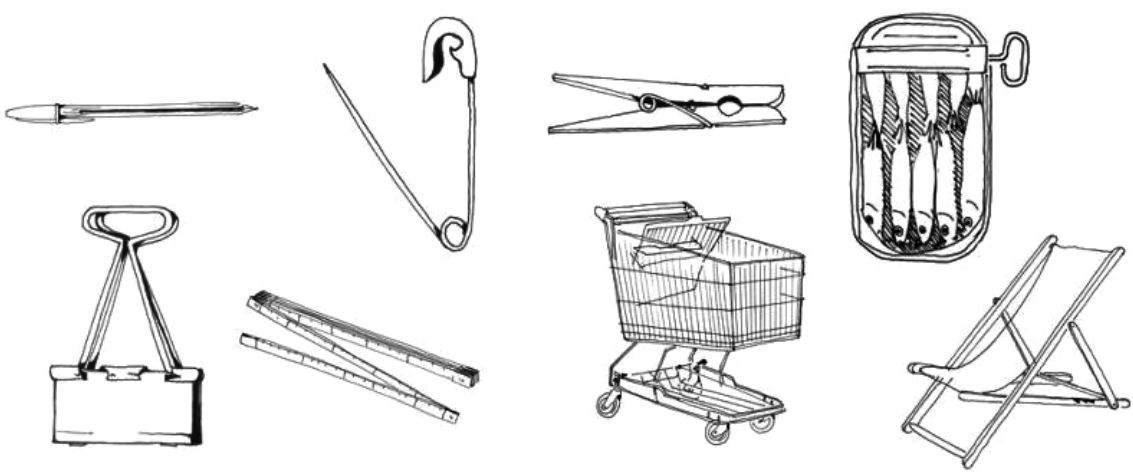

Figura 16. Alcuni artefatti di autori anonimi.

La trasformazione degli oggetti anonimi in oggetti-icone è quindi determinata dall'esperienza pratica e dagli ambienti d'uso. Un esempio: la graffetta non racconta un'epoca, non esprime valori culturali o princìpi ideologici, ma diviene interpretante di un luogo (l'ufficio, la scuola, ...) e delle attività che lì vengono svolte. Questi oggetti-icona in genere non rientrano nella categoria delle icone epocali (come la Fender Stratocaster) o in quella delle icone concettuali (come Futura ed Helvetica), ma si distinguono per essere icone contestuali.

Quando un artefatto è il frutto dell'ingegno di un autore esplicito, si coinvolge l'ambito del design, inteso come progettazione che «opera per sistemi, facendosi carico non solo delle necessità funzionali di uno strumento, ma anche della sua qualità formale ed espressiva» (Branzi 2007: 50). Non sempre, 


\section{Ocula ${ }^{22}$}

Vol 21, No 22 (April 2020) • DOI: 10.12977/ocula2020-21

Anna Riboldi e Salvatore Zingale | Oggetti persistenti. La somiglianza a posteriori nelle icone del design

però, il design precede l'artefatto: al contrario, può accadere che l'oggetto del progetto generi il design. Questo assunto si chiarifica attraverso l'analisi del secondo grado di esistenza dell'autorialità, e nello specifico delle intenzioni che sottendono l'atto progettuale e la sua opera. Infatti, seppure non più anonimo, il progettista di un'icona del design non corrisponde necessariamente alla figura di un "designer", e pertanto la sua spinta ideativa può assumere carattere di spontaneità ed essere priva di una disciplina formale, quindi di un "linguaggio progettuale".

È il caso della Moka Bialetti, che, al pari della Vespa ma per ragioni differenti, si annovera tra le icone dell'italianità (fig. 17). Il suo inventore, Alfonso Bialetti, era un industriale che operava nel ramo della fusione e lavorazione dell'alluminio. Nel 1933, mentre osservava il funzionamento di un particolare tipo di lavatrice (chiamata lisciveuse), per associazione di idee concepì la moka e orientò gran parte della sua produzione verso questo prodotto innovativo, che scalzò rapidamente le tradizionali caffettiere napoletane. Fu solo in seguito, attraverso le strategie commerciali e comunicative del figlio Renato, che la Moka Bialetti assunse il ruolo di icona: la nascita nel 1953 dell'“omino coi baffi" (caricatura di Renato Bialetti firmata Paul Campani) suggellò la relazione inscindibile tra la moka e le abitudini della quotidianità italiana.

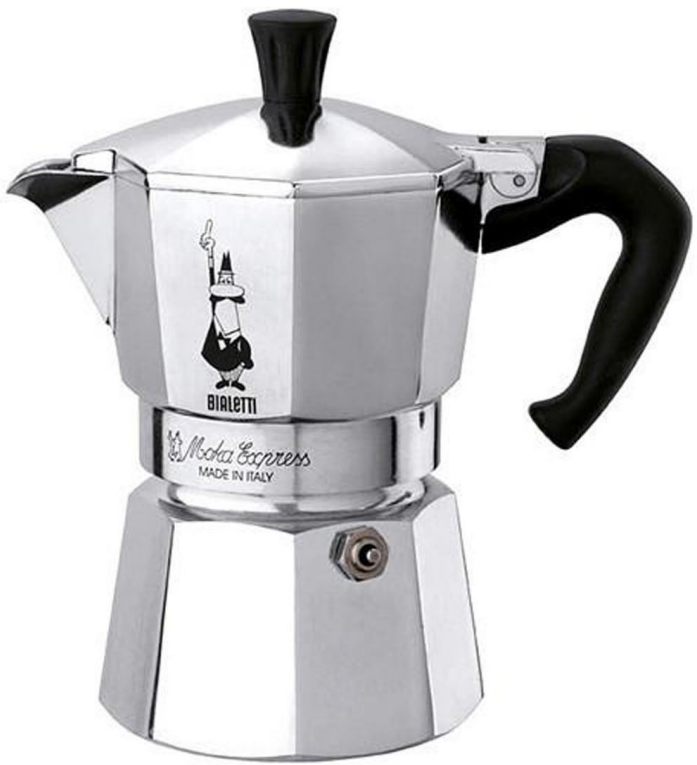

Figura 17. La caffettiera “Moka Express” di Bialetti (1933).

"La Bialetti" (chiamata così per metonimia) costituisce un archetipo dal quale, in seguito, si è evoluto il "design della moka". E, infatti, l'intenzione del suo inventore ha caratteri di libertà e istintività, precede le dinamiche del design, non ha scopi evocativi a priori. Questi ultimi, appunto, vengono acquisiti solo quando l'oggetto entra in relazione con l'immaginario collettivo. 


\section{Ocula ${ }^{22}$}

Vol 21, No 22 (April 2020) • DOI: 10.12977/ocula2020-21

Anna Riboldi e Salvatore Zingale | Oggetti persistenti. La somiglianza a posteriori nelle icone del design
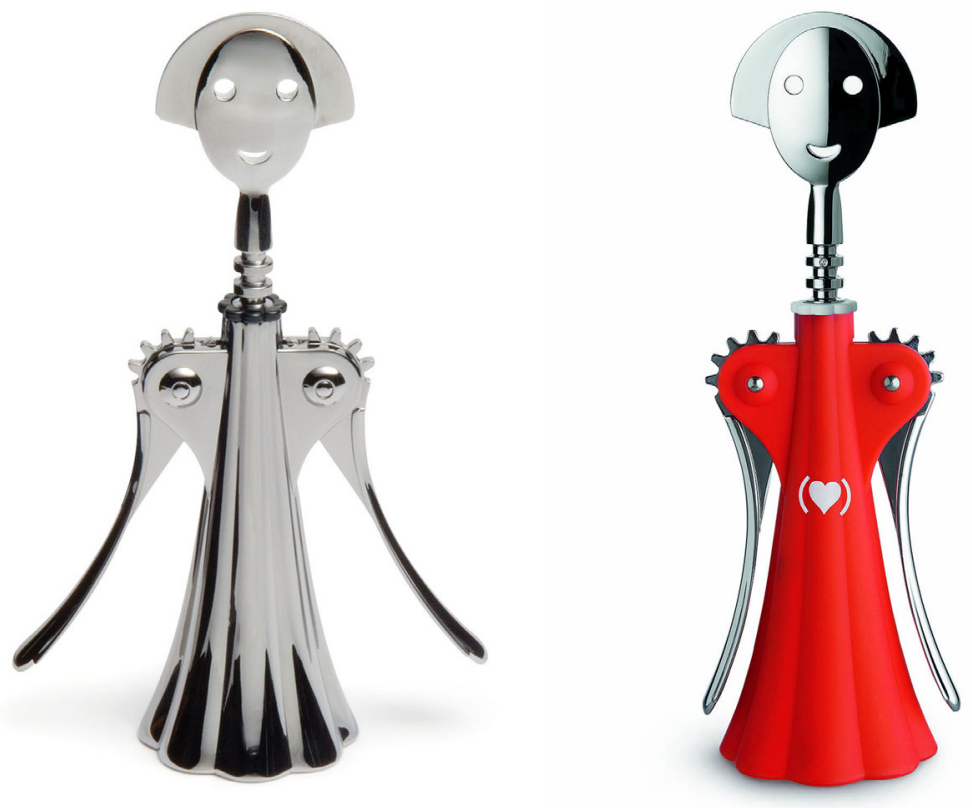

Figura 18. Due versioni del cavatappi “Anna G” di Alessandro Mendini per Alessi (1994).

Si può quindi sostenere che se l'autore di un'icona non è un designer, la progettualità segue l'artefatto, perché è l'uso o l'immaginario che lo riconosce in quanto "progetto"; viceversa, quando l'autore corrisponde alla figura del designer professionista, la progettualità costituisce una conditio sine qua non dell'opera.

Per esempio, in Anna G., l'ormai celeberrimo cavatappi disegnato nel 1994 da Alessandro Mendini per Alessi, si rintraccia quell'iconicità pretestuosa tipica degli artefatti che scaturiscono dai colpi di genio dei designer professionisti (fig. 18). Nato come omaggio ad Anna Gili, progettista che all'epoca frequentava l'atelier di Mendini, questo prodotto è la rappresentazione di una visione postmoderna e giocosa del design. L’iconicità di Anna G. è insita nella pareidolia che tutti sperimentiamo nell'osservare un cavatappi casalingo (il modello dotato di ali d'estrazione e del tipico anello per l'apertura dei tappi a corona): la forma dell'utensile crea in tutti noi un'associazione immediata con la struttura antropomorfa. Il "linguaggio" di Mendini, quindi, è una retorica che si fonda sulla rievocazione di questa illusione pareidolitica e precede la metamorfosi dell'artefatto in icona, poiché costituisce un'intenzione progettuale a priori. C'è da chiedersi allora se l'iconicità di questo tipo di prodotti derivi più dalla notorietà del designer o da questa retorica della somiglianza.

Il discorso sull'autorialità si completa se si esamina l'ultimo grado della sua esistenza: l'intervento di più autori che interpretano il medesimo artefatto (definibile come archetipo). È la condizione descritta dalla storia della lampada direzionabile da tavolo, anche detta "lampada articolata" o "lampada arrendevole”, brevettata nel 1919 dall'ingegnere Curt Fischer, fondatore dell'azienda tedesca di light design Midgard (fig. 19). Questo prodotto affascinò e ispirò 


\section{Ocula}

Vol 21, No 22 (April 2020) • DOI: 10.12977/ocula2020-21

Anna Riboldi e Salvatore Zingale | Oggetti persistenti. La somiglianza a posteriori nelle icone del design

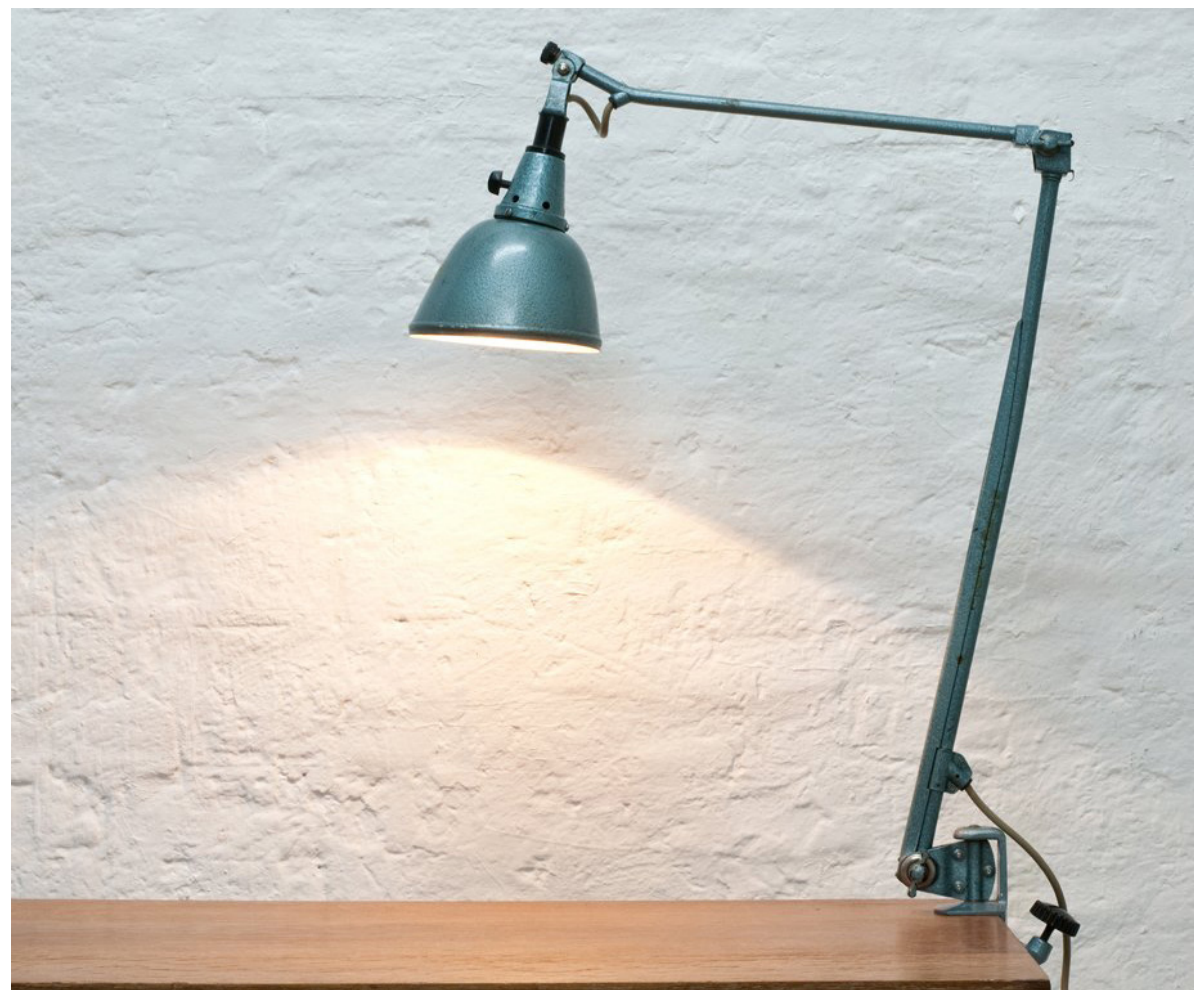

Figura 19. Lampada da scrivania di Curt Fischer per Auma (anni Trenta).

gli architetti Walter Gropius e Marcel Breuer. Il primo, tra il 1927 e il 1931, intraprese rapporti epistolari con Fischer; Breuer provò a disegnare una sua versione del modello. Diversi anni dopo, nel 1972, Richard Sapper concepì per Artemide la lampada Tizio, con il medesimo espediente tecnico (la presenza di un braccio articolato) rielaborato attraverso l'introduzione di contrappesi in acciaio (fig. 20). Quindici anni dopo, Michele De Lucchi e Giancarlo Fassina vinsero il Compasso d'Oro con la lampada Tolomeo, disegnata nel 1987 sulla base degli stessi princìi, sempre per Artemide (fig. 21).

Tutte le reinterpretazioni citate custodiscono un valore iconico, perché i loro attributi di senso non possono essere ridotti a quelli del primo modello di lampada articolata. Queste icone del design costituiscono riscritture del medesimo oggetto. Ne consegue che il susseguirsi delle declinazioni di un artefatto rafforza il valore iconico dell'archetipo da cui esse derivano: la copia, in questo senso, non è da considerarsi al pari di una mera imitazione, bensì come un'interpretazione consacrante.

\section{Conclusione: ci saranno altre icone?}

Una preoccupazione che tormenta chi si occupa di progetto (cfr. Alessi 2018) concerne il futuro delle icone. Si è maturata infatti la sensazione di una scarsità di nuove referenze, come se il design non fosse più in grado di concepire artefatti capaci di scuotere la nostra immaginazione collettiva. 


\section{Ocula $^{22}$}

Vol 21, No 22 (April 2020) • DOI: 10.12977/ocula2020-21

Anna Riboldi e Salvatore Zingale | Oggetti persistenti. La somiglianza a posteriori nelle icone del design

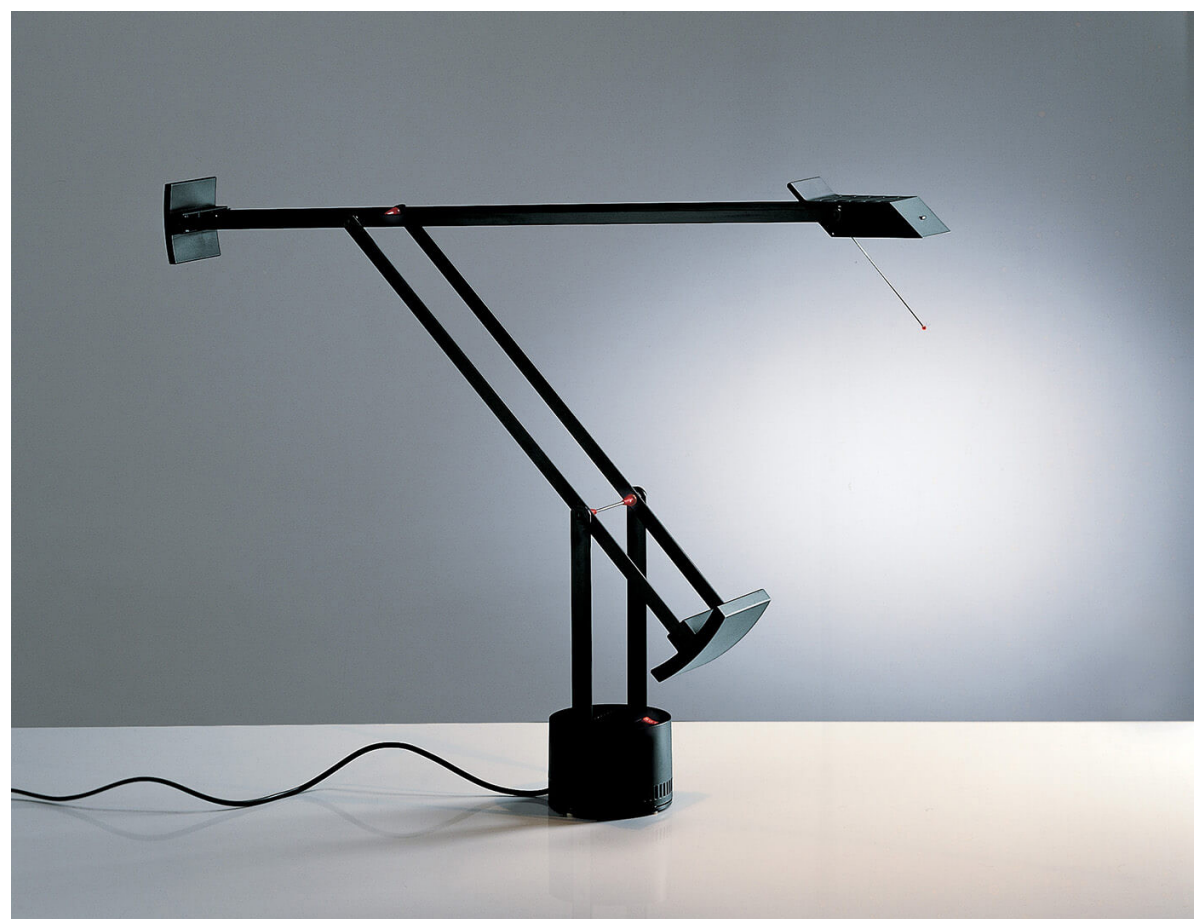

Figura 20. Lampada “Tizio" di Richard Sapper per Artemide (1972).

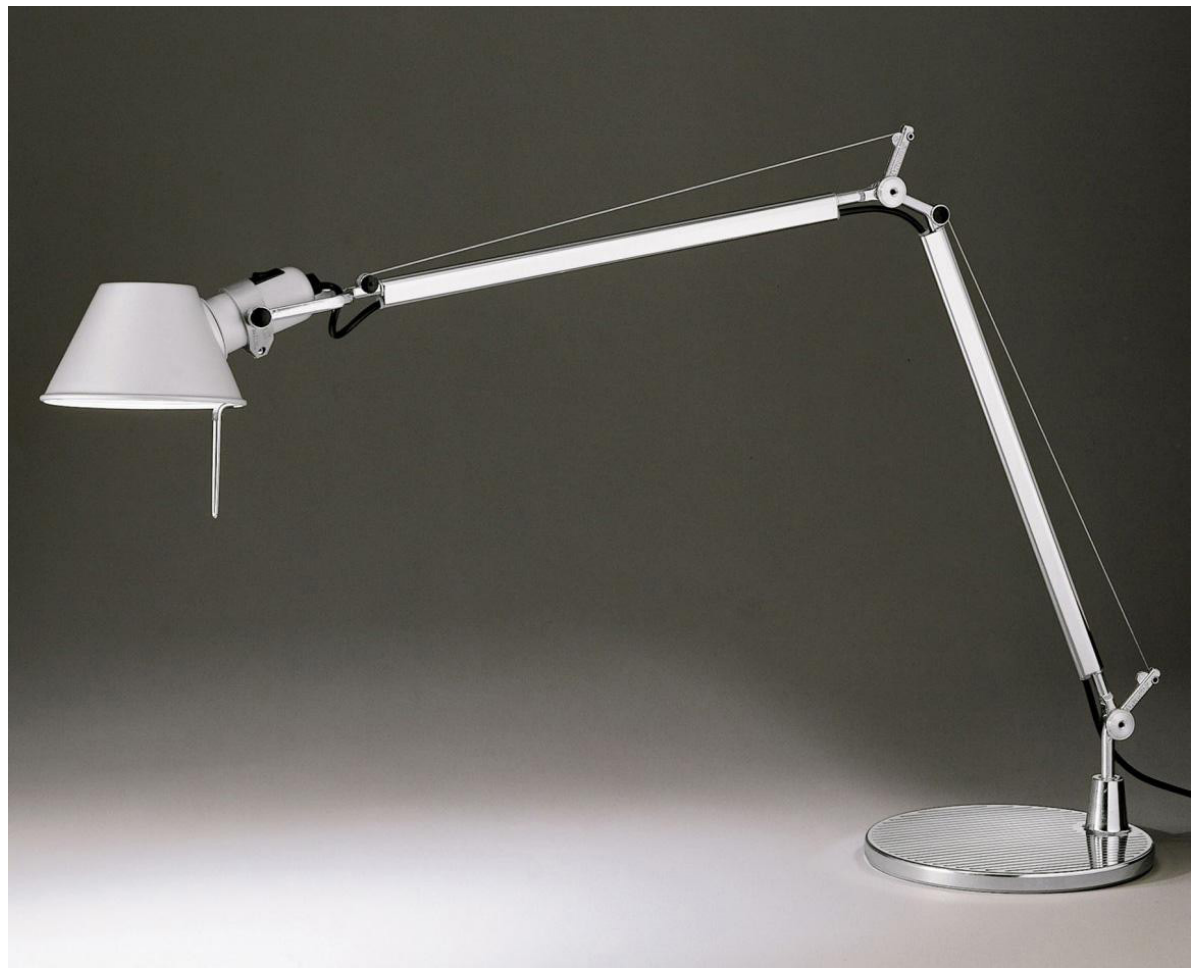

Figura 21. Lampada “Tolomeo" di Michele De Lucchi e Giancarlo Fassina per Artemide (1987). 


\section{Ocula}

Vol 21, No 22 (April 2020) • DOI: 10.12977/ocula2020-21

Anna Riboldi e Salvatore Zingale | Oggetti persistenti. La somiglianza a posteriori nelle icone del design

Sotto certi aspetti, ciò può essere verosimile: le icone invecchiano, ma non scadono. Anzi, con il tempo si affinano e acquistano valore, perché ambiscono all'eternità. La loro sempreverde presenza, stratificata in anni di percorsi inventivi, ha dato origine a un sovraffollamento iconico che lascia poco spazio ai nuovi oggetti del progetto. È però altrettanto evidente, come argomentato, che più riedizioni di un medesimo artefatto possono diventare icone senza sovrapporsi e compromettersi a vicenda. Al contrario, le stesse innescano una rete di riferimenti reciproci che rafforza il valore simbolico di tutte le versioni.

Quindi, sebbene sia superata l'epoca delle grandi invenzioni formali e si sia entrati in quella della variazione e della reinterpretazione, ciò non è sufficiente a limitare la genesi di nuove icone del design. Come si è ripetuto, il processo di conferimento di valori simbolici a un artefatto è innescato da più fattori, i quali interagiscono e a volte creano la giusta alchimia (il carattere dell'epoca storica, l'immaginario del tempo, le tecnologie produttive, le qualità funzionali e così via). Per prevedere con larga approssimazione il destino degli artefatti di concezione odierna è quindi opportuno soffermarsi sulle qualità dell'epoca che stiamo attraversando.

Nello specifico, si è visto come la contemporaneità sia determinata da un flusso indefinito e in convulso aggiornamento di avvenimenti, dei quali si ha elevata informazione e scarsa cognizione. Lo stesso carattere discreto del tempo è riprodotto in un immaginario collettivo che, forse, non è più così condiviso, ma è atomizzato dall'aumento dei canali e, di conseguenza, dalle possibilità di accesso alla realtà, o alle realtà. Il concetto di "pubblico" perde il suo ruolo, perché i percorsi di ciascun utente si differenziano, districandosi in una complessità pressoché infinita di proposte e di scelte. Anche qui, sfumano tutte le coordinate indispensabili alla formazione di un nuovo valore simbolico comunemente riconosciuto.

Sul piano della tecnica e della produzione degli artefatti, l'approccio con il quale oggi si sviluppano i progetti è orientato a una persistenza nell'immediato, non a lungo termine, degli artefatti, sempre nella prospettiva del costante rinnovamento propria del consumismo. Questa visione del progetto è degenerata nella cosiddetta "obsolescenza programmata", strategia che mira al veloce deperimento di un prodotto e che certo non si accorda con le ambizioni pervasive dell'icona nella storia.

Oltre a non essere particolarmente longevi, gran parte dei nuovi prodotti del design dialogano con una rete di servizi digitali con cui comunicano e cooperano per il conseguimento dei propri obiettivi d'uso. Questi artefatti, quindi, subiscono una frammentazione tra la propria consistenza fisica (plastica o grafica) e la funzione d'uso, della quale vengono deprivati.

Gli oggetti che ci circondano nell'epoca dell'elettronica hanno perso gran parte di quella totale autonomia che li caratterizzava nel xx secolo. Ciascuno di essi vive come link di una rete, come parte di un sistema informatico che attraversa la città e ne rende liquide le antiche separazioni funzionali. L'oggetto informatico, il computer, ha rotto definitivamente anche quel rapporto tra forma e funzione che alimentava l'identità compatta dell'oggetto moderno; esso non ha più una ma tante funzioni, quante sono le 


\section{Ocula}

Vol 21, No 22 (April 2020) • DOI: 10.12977/ocula2020-21

Anna Riboldi e Salvatore Zingale | Oggetti persistenti. La somiglianza a posteriori nelle icone del design

necessità di ogni singolo operatore. Gli oggetti sono piuttosto (come dicono i teorici) dei funzionoidi, cioè realtà intelligenti ma portatrici di una identità sfuggente, indeterminata, che crea un alone opaco intorno alla loro lucida efficienza. (Branzi 2007: 276)

La contemporaneità, nelle sue molteplici variabili, ha determinato uno stravolgimento del nostro rapporto con gli artefatti, tanto che questo tipo di analisi potrebbe scoraggiare la formulazione di previsioni ottimistiche sulla nascita di nuove icone del design. Tutto ciò fino a sfiorare il pensiero che il concetto stesso di icona sia anch'esso un'icona, erede di un'epoca trascorsa, pertanto anacronistica e non più coerente con la realtà attuale (cfr. Alessi 2018).

Nell'ambito della teoria e della critica del design, tale argomento aprirebbe un interessante percorso di indagine e di riflessione, finalizzato a proporre nuove categorie, più coerenti con l'epoca attuale e con le qualità degli oggetti funzionoidi di cui parla Andrea Branzi. Tali categorie dovrebbero permettere una visione dei prodotti del design liberata dall'ossessione iconica che agita l'ansia di creatività di molti designer. Oggi al design spettano nuove imprese: non più allettare l'immaginario di utenti e consumatori, ma proporre attraverso l'artefatto stimoli per l'azione, modelli d'uso consapevole, contatti dialogici.

La nozione di icona associata al design, come abbiamo visto, ha un valore oscillante: da un lato un'icona si pone come interpretante dei valori di un'epoca, dall'altra non si libera dal retaggio di inviolabilità delle immagini bizantine. Se così la nozione di icona finora analizzata ci porta a vedere alcuni "pezzi classici" del design come avvolti da una sorta di sacra ed eterna staticità - come oggetti intoccabili e persistenti -, la rottura di tale concezione porta a individuare nell'opera del design altri scenari. Da oggetti chiusi nella propria immagine, gli artefatti diventano ora portatori di valori possibili e relazionali. "Possibili", perché capaci di aprire squarci su inedite concezioni della realtà e su nuove modalità d'uso. "Relazionali", perché capaci di tracciare connessioni con altri artefatti e di favorire utenze partecipate, proiettando la funzione dell'artefatto in una rete di condivisioni sempre più ampia, eterogenea, pervasiva.

Perché, come ben ricorda Chiara Alessi, il ruolo sociale di un progetto è indipendente dal suo divenire o non divenire icona.

\section{Bibliografia}

Alessi, Chiara

2018 Le caffettiere dei miei bisnonni. La fine delle icone nel design italiano, Torino, Utet.

Barthes, Roland

1957 Mythologies, Paris, Seuil (tr. it., Miti d’oggi, Torino, Einaudi, 1974).

Baudrillard, Jean

1968 Le Système des objets : la consommation des signes, Paris, Gallimard (tr. it. Il sistema degli oggetti, Firenze, Giunti, 1968). 


\section{Ocula ${ }^{22}$}

Vol 21, No 22 (April 2020) • DOI: 10.12977/ocula2020-21

Anna Riboldi e Salvatore Zingale | Oggetti persistenti. La somiglianza a posteriori nelle icone del design

Bauman, Zygmunt

2000 Liquid Modernity, Cambridge, Polity (tr. it. Modernità liquida, Roma-Bari, Laterza, 2012).

Bettetini, Maria

2006 Contro le immagini. Le radici dell’iconoclastia, Roma-Bari, Laterza.

Bonfantini, Massimo; Renzi, Emilio (a cura di)

2001 Oggetti Novecento, Bergamo, Moretti \& Vitali. Seconda edizione: Oggetti Novecento Duemila, Milano, ATì, 2011.

Branzi, Andrea

2007 Capire il design, Firenze, Giunti.

Bringhurst, Robert

1992 The Elements of Typographic Style, Vancouver, Hartley \& Marks Publishers Inc. (tr. it. Gli elementi dello stile tipografico, Milano, Sylvestre Bonnard, 2001).

Dri, Pietro

1994 Serendippo, Roma, Editori Riuniti.

Eco, Umberto

1975 Trattato di semiotica generale, Milano, Bompiani.

Fabbrichesi Leo, Rossella

1983 La polemica sull'iconismo, Napoli, Esi.

Fadda, Emanuele

2013 Peirce, Roma, Carocci.

Genette, Gérard

1987 Seuils, Paris, Editions du Seuils (tr. it. Soglie. I dintorni del testo, Torino, Einaudi, 1989).

Greimas, Algirdas J.

1984 "Sémiotique figurative et sémiotique plastique”, Actes Sémiotiques.

Documents, 60, Paris, (tr. it. "Semiotica figurativa e semiotica plastica", tr. it. in Corrain, Lucia e Valenti, Paolo, a cura di, Leggere l'opera d'arte. Dal figurativo all'astratto, Bologna, Esculapio, 2002, pp. 33-51).

Greimas, Algirdas J.; Courtés, Joseph

1979 Sémiotique. Dictionnaire raisonné de la théorie du langage, Paris, Hachette (tr. it. Semiotica. Dizionario ragionato della teoria del linguaggio, a cura di Paolo Fabbri, Milano, Bruno Mondadori, 2007).

Hochuli, Jost

2015 Das Detail in der Typografie, Paris, Éditions B4 (tr. it. Il dettaglio in tipografia, Milano, Lazy Dog, 2018).

Kitzinger, Ernst

1954 "The Cult of Images in the Age before Iconoclasm", Dumbarton Oaks Papers, 8, pp. 83-150 (tr. it. Il culto delle immagini. L'arte bizantina dal cristianesimo delle origini all'iconoclastia, Roma, Meltemi, 2018).

Maffesoli, Michel

2008 Iconologies. Nos idol@tries postmodernes, Paris, Éditions Albin Michel (tr. it. Icone d’oggi. Le nostre idol@trie postmoderne, Palermo, Sellerio, 2009). 


\section{Ocula}

Vol 21, No 22 (April 2020) • DOI: 10.12977/ocula2020-21

Anna Riboldi e Salvatore Zingale | Oggetti persistenti. La somiglianza a posteriori nelle icone del design

Manzini, Ezio

2018 Politiche del quotidiano: Progetti di vita che cambiano il mondo, Roma, Edizioni di Comunità.

Merton, Robert K.; Barber, Elinor G.

2004 The Travels and Adventures of Serendipity: A Study in Sociological Semantics and the Sociology of Science, Princeton, Princeton University Press (tr. it. Viaggi e avventure della Serendipity. Saggio di semantica sociologica e sociologia della scienza, Bologna, il Mulino, 2008).

Munari, Bruno

1981 Da cosa nasce cosa. Appunti per una metodologia progettuale, Roma-Bari, Laterza.

Peirce, Charles S.

[CP] Collected Papers, voll. I-VI a cura di Ch. Harshome e P. Weiss, 1931-1935; voll. VII-VIII a cura di A. Burks, 1958; Cambridge MA, Harward University Press (tr. it. parziale Id. Opere, a cura di M.A. Bonfantini, con la collaborazione di G. Proni, Milano, Bompiani, 2003).

Ponzio, Augusto

1976 La semiotica in Italia. Fondamenti teorici, Bari, Dedalo.

Proni, Giampaolo

2017 La semiotica di Charles S. Peirce. Il sistema e l'evoluzione, Roma, Aracne.

Spaziante, Lucio

2016 Icone pop. Identità e apparenze tra semiotica e musica, Milano, Bruno Mondadori.

Velmans, Tania

2000 Icone. Il Grande Viaggio, Milano, Jaka Book.

Vitta, Maurizio

1996 Il disegno delle cose. Storia degli oggetti e teoria del design, Napoli, Liguori.

1999 Il sistema delle immagini. Estetica della rappresentazione quotidiana, Napoli, Liguori.

Zingale, Salvatore

2012 Interpretazione e progetto. Semiotica dell'inventiva, Milano, FrancoAngeli.

Anna Riboldi è laureata in Design della comunicazione alla Scuola del Design del Politecnico di Milano, dove attualmente svolge attività di ricerca e collabora al corso Teorie della comunicazione.

Salvatore Zingale, Professore associato, insegna Semiotica del progetto e Teorie della comunicazione alla Scuola del Design del Politecnico di Milano. Autore di saggi sulla semiotica dei processi progettuali e sul design, si interessa in particolare di pensiero inventivo, dialogicità e di culture visuali. 